\title{
PREMIÈRE CONTRIBUTION A L'ÉTUDE DES TEIGNES EN HAUTE VOLTA. REVUE DES DERMATOPHYTIES ET DES DERMATOPHYTES EN AFRIQUE NOIRE
}

\author{
Par J. Biguet, S. Deblock, G. Cochet et P. OUADRaogo
}

En 1956 et 1957, grâce à l'un de nous (1), notre laboratoire a disposé de 215 prélèvements effectués sur le cuir chevelu d'enfants suspects de teigne en Haute-Volta où, à notre connaissance du moins, aucun travail concernant ces affections n'avait été publié jusqu'ici. A l'occasion de l'étude de ce matériel, nous avons pensé utile de faire une revue critique des dermatophyties et des dermatophytes en Afrique Noire, en insistant tout particulièrement sur les teignes et leurs agents, de façon à ce que les recherches effectuées ultérieurement puissent bénéficier des indications fournies par cette mise au point.

\section{Les teignes en Haute Volta}

La Haute-Volta (carte C) (2), située au centre de l'Afrique Occidentale Française, a l'aspect d'un plateau qui couvre une superficie de $316.000 \mathrm{~km}^{2}$; elle est constituée essentiellement par le bassin de la Volta supérieure.

Son climat est généralement celui de la zone soudanienne avec trois saisons principales, mais, exception faite de l'hivernage, le climat est dans l'ensemble ensoleillé et sec, avec des variations sensibles de température.

La population, de 3.070 .000 habitants, est, avec sa densité de 10 habitants au $\mathrm{km}^{2}$, l'une des plus fortes de l'A.O.F. Les deux plus

(1) Une première série de prélèvements a été personnellement effectuée par P. Ouadraogo en 1956 ; il nous a procuré la seconde série en 1957 par l'interniédiaire de son cousin, M. Charles Gnimigna Ouadraogo, que nous remercions infiniment de la précieuse collaboration qu'il nous a ainsi apportée.

(2) Nous empruntons les renseignements qui suivent à une brochure éditée par le Service d'Information et de Documentation du Ministère de la France d'Outrc-Mer, qui a eu l'amabilité de nous fournir une précieuse documentation sur l'Afrique Noire. 
grandes villes sont Ouagadougou, la capitale, et Bobo-Dioulasso, qui est un centre commercial. Le groupe ethnique le plus important est constitué par des races voltaïques, très anciennement établies dans le pays, dont l'élément déterminant, tant par le nombre (2.600.000) que par la structure sociale et politique, est formé par les Mossi, à còté desquels on pourrait citer entre autres les Bobo (273.000). En dehors de ces populations fétichistes existent des groupes attachés à l'islamisme (Pheul, Senougo, etc...), qui comprennent environ 550.000 personnes. Alors que la masse de la population se consacre à l'agriculture, les groupes islamisés s'adonnent plus volontiers au commerce et à l'élevage. Dans l'ensemble, en Haute-Volta, comme presque partout ailleurs en Afrique, l'alimentation des autochtones est carencée en protéines.

\section{Formes cliniques et fréauence relative des teignes en Haute-Volta}

Les prélèvements que nous avons étudiés étaient au nombre de 215 , dont 65 provenaient de Ouagadougou et des villages environnants et 150 de la région de Bobo-Dioulasso. Les premiers avaient intéressé des enfants qui, en majorité, étaient des Mossi (46) (3), les seconds, des enfants presque tous de race Bobo. Les jeunes patients ont été vus dans des consultations de dispensaires en octobre 1956 et 1957.

Le diagnostic de teigne fut confirmé pour 171 d'entre eux par l'examen direct des cheveux ou leur culture sur milieu de Sabouraud. Il s'agissait de 148 Trichophyties (86,54\%), de 21 Microspories $(12,28 \%)$ et de deux cas mixtes. Précisons tout de suite qu'à l'examen microscopique, ces deux derniers cas avaient été étiquetés «Trichophytie » et que, seule, la culture, a révélé la Microsporie associée. Mais il convient d'ajouter que les cheveux étaient généralement peu nombreux par prélèvement, ce qui nous incitait à restreindre l'examen direct à un ou deux fragments, le reste du matériel étant ensemencé. Quant au Favus, il n'existe pas ou est rare dans les deux régions prospectées, puisque nous n'en avons dépisté aucun cas.

A Ouagadougou et dans ses environs, sur 63 prélèvements positifs, nous avons observé 53 Trichophyties, 9 Microspories et un cas mixte (soit respectivement $84,12 \%, 14,28 \%$ et $1,58 \%$ ), tandis que, dans la région de Bobo-Dioulasso, 108 teigneux se répartissaient en

(3) En dehors des Mossi, des prélèvements tous positifs furent effectués sur 7 enfants Bobo, 5 Boussansi, 2 Pheul, 2 Gouronsi, 1 Haoussa, 1 Samogo, 1 Dioula 


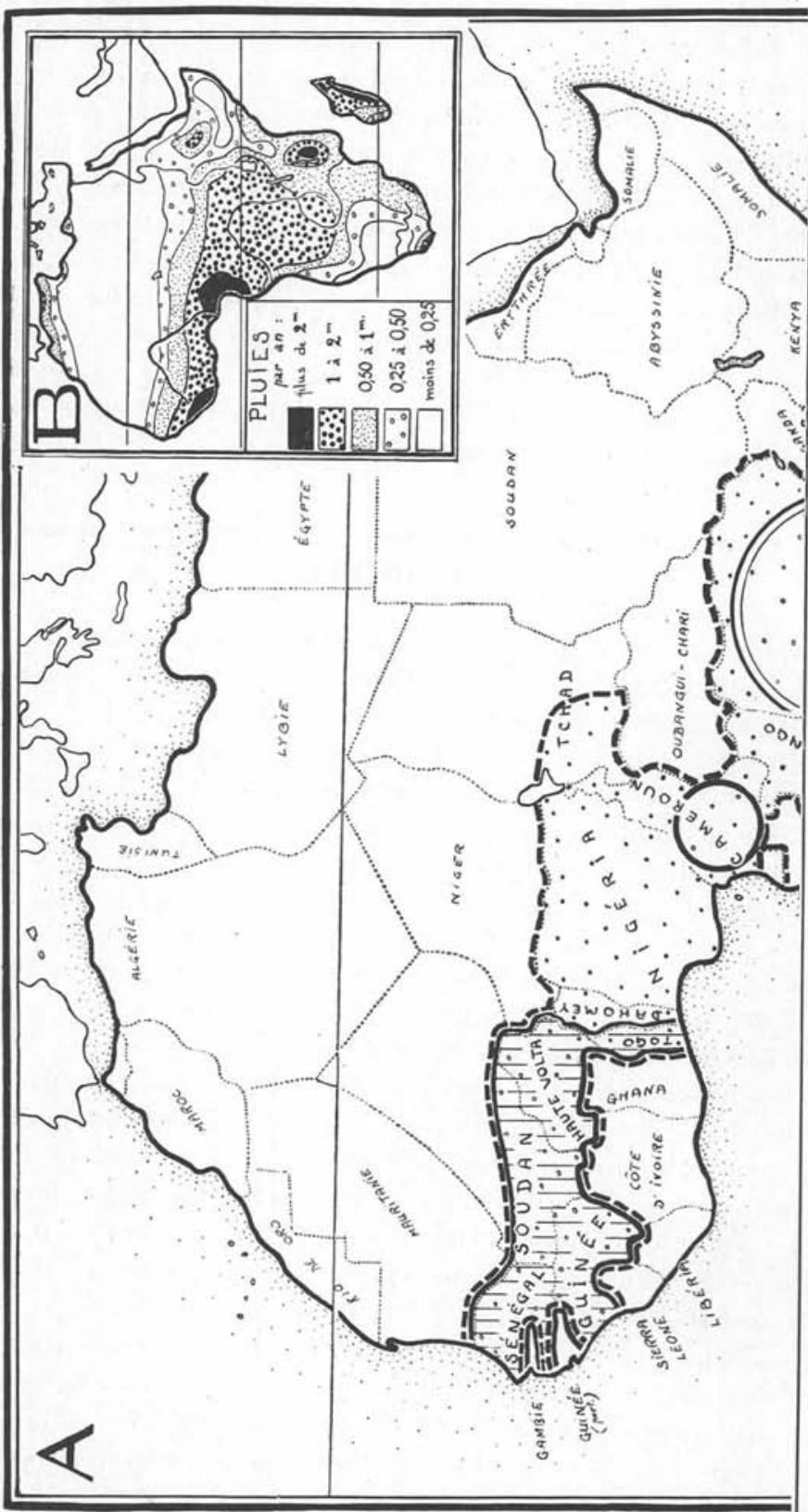




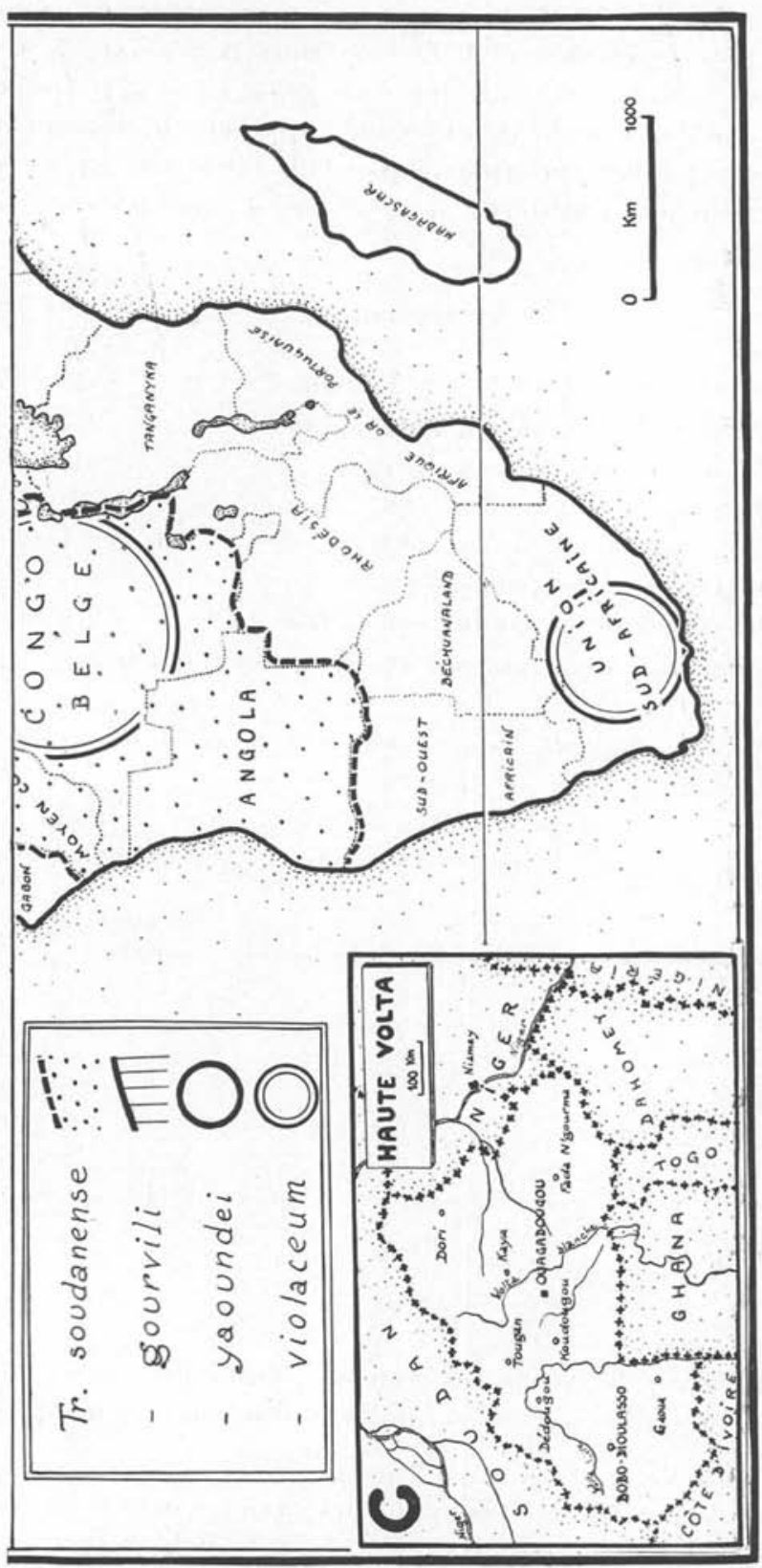


95 Trichophyties, 12 Microspories et un cas mixte (soit respectivement $87,96 \%, 11,11 \%$ et $0,92 \%)$. Sous la réserve de recherches ultérieures portant sur un nombre plus élevé d'individus, il ne semble donc pas y avoir de différence appréciable dans la fréquence relative des teignes microsporiques et trichophytiques dans les deux territoires considérés que séparent quelques centaines de kilomètres.

\section{Mycologie}

Nous avons au total isolé 109 souches :

Trichophyton gourvili Catanei, $1933 \ldots \ldots .64$ fois

T. soudanense Joyeux, $1912 \ldots \ldots \ldots \ldots \ldots .23$ fois

T. rubrum (Castellani, 1909) .......... 2 fois

T. tonsurans Malmsten, $1845 \ldots \ldots \ldots \ldots \ldots 1$ fois

T. ferrugineum $(\mathrm{Ota}, 1922) \ldots \ldots \ldots \ldots \ldots .4$ fois

Microsporum audouini Gruby, $1843 \ldots \ldots \ldots 15$ fois

Par région, nos résultats ont été résumés dans le tableau I.

TABleau I. - Souches isolées dans les deux régions prospectées

\begin{tabular}{|c|c|c|c|c|}
\hline \multirow[t]{2}{*}{ ESPÈCES } & \multicolumn{2}{|c|}{$\begin{array}{c}\text { RÉGION } \\
\text { DE OUAGADOUGOU }\end{array}$} & \multicolumn{2}{|c|}{$\begin{array}{cc}\text { RÉgION } \\
\text { DE }\end{array}$} \\
\hline & Nombre & $\%$ & Nombre & $\%$ \\
\hline T. gourvili ....... & 34 & 68 & 30 & 50,84 \\
\hline T. soudanense .... & 8 & 16 & 15 & 25,42 \\
\hline T. rubrum ....... & & & 2 & 3,39 \\
\hline T. tonsurans ..... & & & 1 & 1,69 \\
\hline T. ferrugineum .... & 4 & 8 & & \\
\hline M. audouini ...... & 4 & 8 & 11 & 18,64 \\
\hline Total ...... & 50 & & 59 & \\
\hline
\end{tabular}

La fréquence relative de ces souches, leur étude et les conditions de leur isolement nous ont conduits à quelques remarques :

$1^{\circ}$ En Haute-Volta, T. gourvili constitue, dans les limites de notre expérience, l'agent le plus commun des Trichophyties puisque nous l'avons identifié trois fois plus souvent que $T$. soudanense. T. gourvili, aux belles colonies en rosace d'un violet intense le plus souvent frangé de blanc, est peu polymorphe. Parfois cependant certaines de ces cultu- 
res glabres ou pigmentées ressemblaient beaucoup à $T$. violaceum; mais leur morphologie microscopique très différente permettait aisément de rectifier la diagnose.

$2^{\circ} T$. soudanense, contrairement à l'espèce précédente, montrail un polymorphisme considérable, que nous avions déjà remarqué antérieurement pour les souches camerounaises (Cochet et coll., 1957). Précisons que de nombreuses colonies présentaient des hyphtes aleurifères typiques et que plusieurs de nos souches sont devenues vioiettes en vieillissant.

$3^{\circ}$ Toujours en ce qui concerne les espèces qui déterminent des Trichophyties, nous remarquons l'isolement dans la région de Bobo-Dioulasso de deux souches de $T$. rubrum et d'une souche de $T$. tonsurans, espèces cosmopolites, dont la première s'isole plus habituellement des lésions de la peau dite glabre. Les souches de T. rubrum, bien pigmentées en rouge violacé, étaient très duveteuses et riches en aleuries : nous n'avons pas constaté la formation de fuseaux; elles étaient moculables au cobaye (lésion néo-endothrix du poil).

$4^{\circ}$ Nous noterons la présence de $T$. ferrugineum dans les Microspories; ce champignon parait peu fréquent en Haute-Volta où il fut observé seulement dans la région de Ouagadougou. Quant aux souches de $M$. audouini, ia plupart étaient assez semblables macroscopiquement à celles que nous avions isolées antérieurement au Cameroun (Cochet et coll., 1957), et ordinairement plus vivaces que les souches métropolitaines; elles étaient duveteuses et parfois rosées. Quelques-unes d'entre elles cependant étaient de culture nettement plus lente et n'atteignaient jamais au développement blanc superficiel des autres; elles poussaient en effet surtout dans la profondeur de la gélose et, sur riz, cultivaient beaucoup plus pauvrement que les souches duveteuses, dont le comportement se rapprochait de $M$. canis; mais, macroscopiquement, quels que soient la souche et le milieu de culture considérés, les fuseaux étaient absents ou peu fréquents. Ajoutons enfin que les colonies duveteuses furent inoculées avec succès au cobaye (4).

$5^{\circ}$ Enfin, notre attention a été attirée par un certain nombre de cas mixtes où se trouvaient associés sur une même tête deux dermatophytes différents, à savoir :
trois fois $T$. gourvili et $T$. soudanense,
une fois $T$. gourvili et $T$. ferrugineum,
une fois $T$. soudanense et $M$. andouini.

Il est vraisemblable que pareilles associations eussent été plus fréquemment notées si nous avions disposé pour l'ensemencement d'un matériel plus abondant.

(4) L'inoculation des souches à développement superficiel réduit est en cours ; en fait, les souches de ces deux types de Microsporum, ainsi que toutes les autres que nous avons reçues des autres territoires d'Afrique Noire font actuellement l'objet d'une étude d'ensemble la plus complète possible (en collaboration avec G. Duc), étude dont la nécessité ne nous est apparue qu'assez tardivement en lisant les divers auteurs qui ont traité des Microsporum africains. 


\section{Revue des dermatophyties et des Dermatophytes en Afrique Noire}

L' "Afrique Noire » est une entité ethnique dont les limites géographiques sont naturellement imprécises en raison de l'hétérogénéité de la population des divers territoires et des métissages qui se sont produits au cours des siècles entre races blanches et races noires. Nous rassemblons cependant sous cette appellation les pays africains qui sont situés au Sud des déserts limités approximativement par le parallèle qui passe par St-Louis-du-Sénégal. De cet ensemble, nous excluons l'Erythrée et les Somalies, de population blanche, ainsi que l'Ethiopie, parce que, dans la race éthiopienne, les caractères négroïdes sont très atténués. Cette dernière exclusion est d'ailleurs peut-être beaucoup plus discutable, mais elle est sans répercussion pratique, puisque nous n'avons trouvé aucun document intéressant ce pays. Les cartes jointes rappellent la division territoriale (A) et évoquent, en fonction des chutes de pluie, les très grandes zones climatiques (B).

Le problème des dermatophyties et des dermatophytes en Afrique Noire a été abordé dans des territoires variés et sous des angles divers par un certain nombre d'auteurs, au premier rang desquels nous retiendrons Catanei et Vanbreuseghem. Le premier cité, dont les travaux ont tant contribué à la connaissance des teignes en Afrique du Nord, a écrit, en 1939, plusieurs articles qui faisaient le point de son expérience personnelle sur les teignes et leurs champignons en Afrique et dans les autres colonies françaises; le second, depuis une dizaine d'années, a réalisé au Congo Belge un travail qui n'a d'équivalent nulle part ailleurs en Afrique Noire. Afin de ne pas alourdir notre exposé, nous nous contenterons de résumer en un tableau (tableau II) les publications que nous avons lues et qui traitaient des dermatophyties et des dermatophytes en Afrique Noire, et nous nous efforcerons seulement de les commenter et de faire la synthèse des renseignements qu'elles nous apportent.

La première remarque qui s'impose d'ailleurs est la nette insuffisance de notre documentation, très souvent fragmentaire, parfois inexistante; il est nécessaire, en particulier, de noter que, sauf 


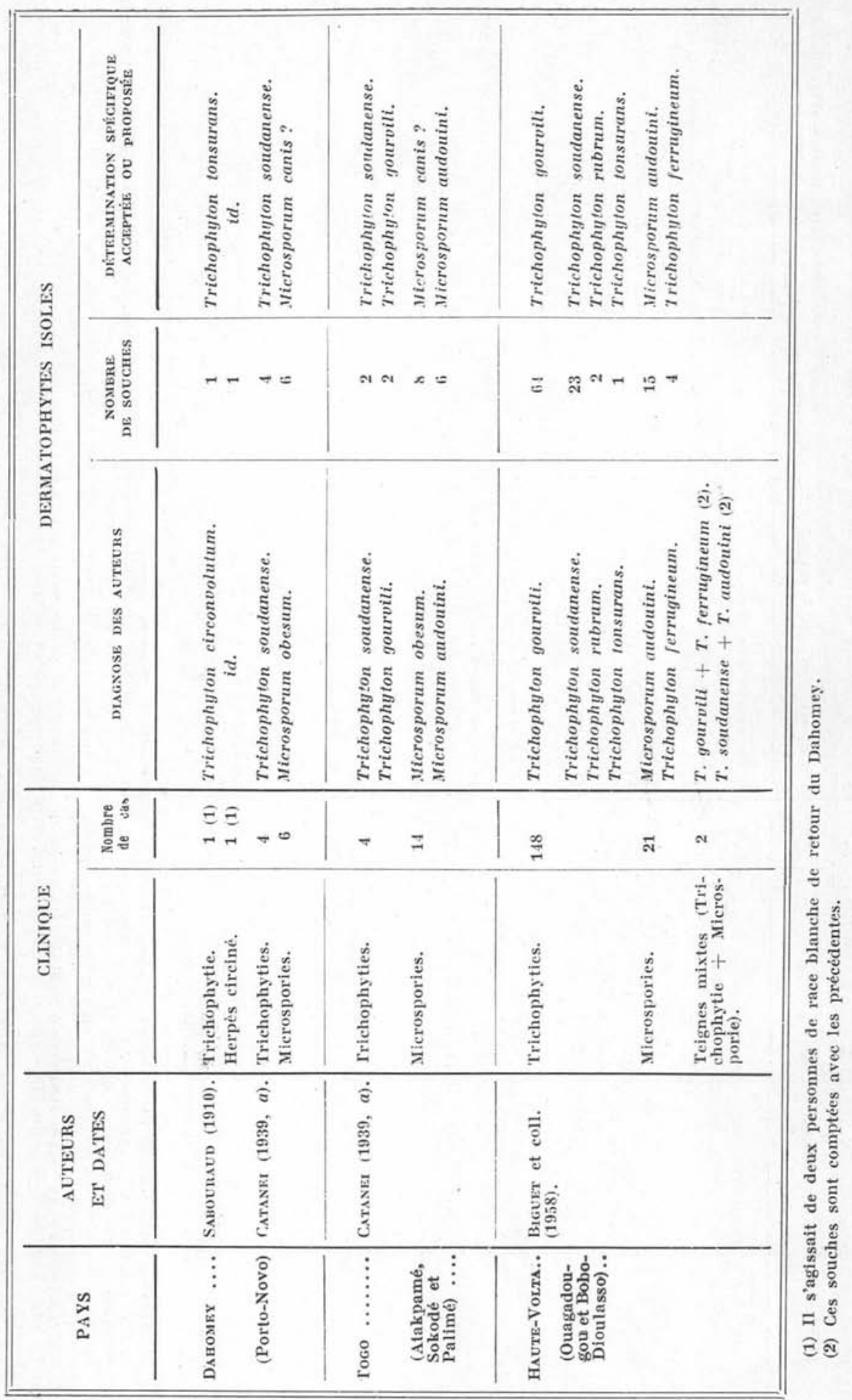




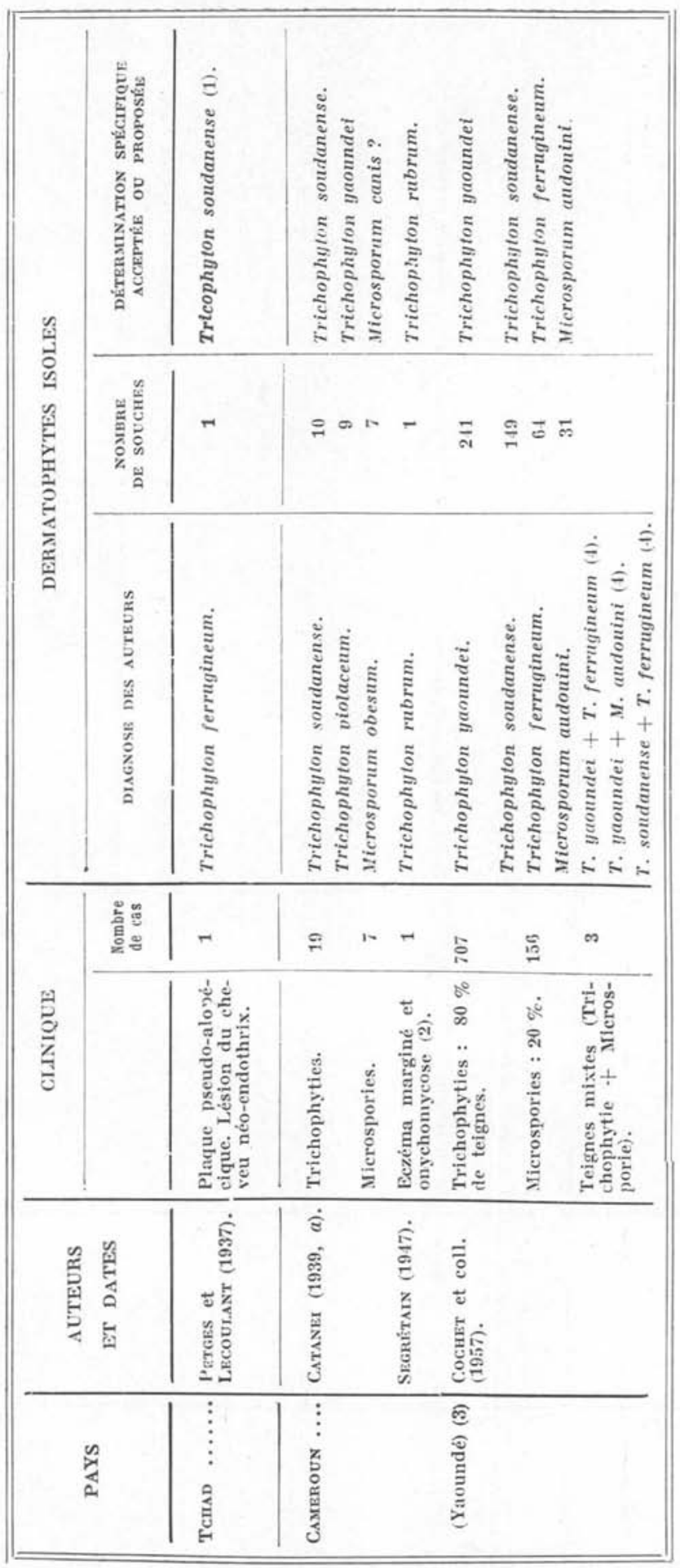

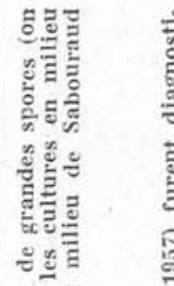

进卷言

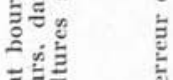

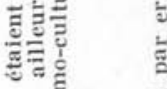

关就

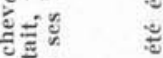

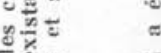

$\because \tilde{c}=$

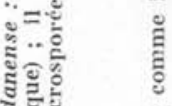

踣

计影范

ป⿻ํㅀ हू

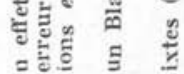

造

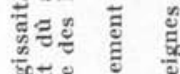

प듕 छ

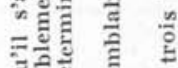

틀

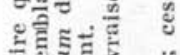

แ

的的政

远踏

팔 =

하뭉

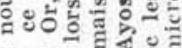

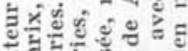

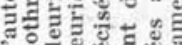

-

泮

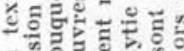

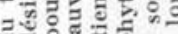

궁

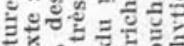

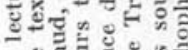

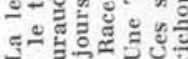

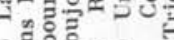

ิ

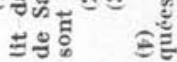




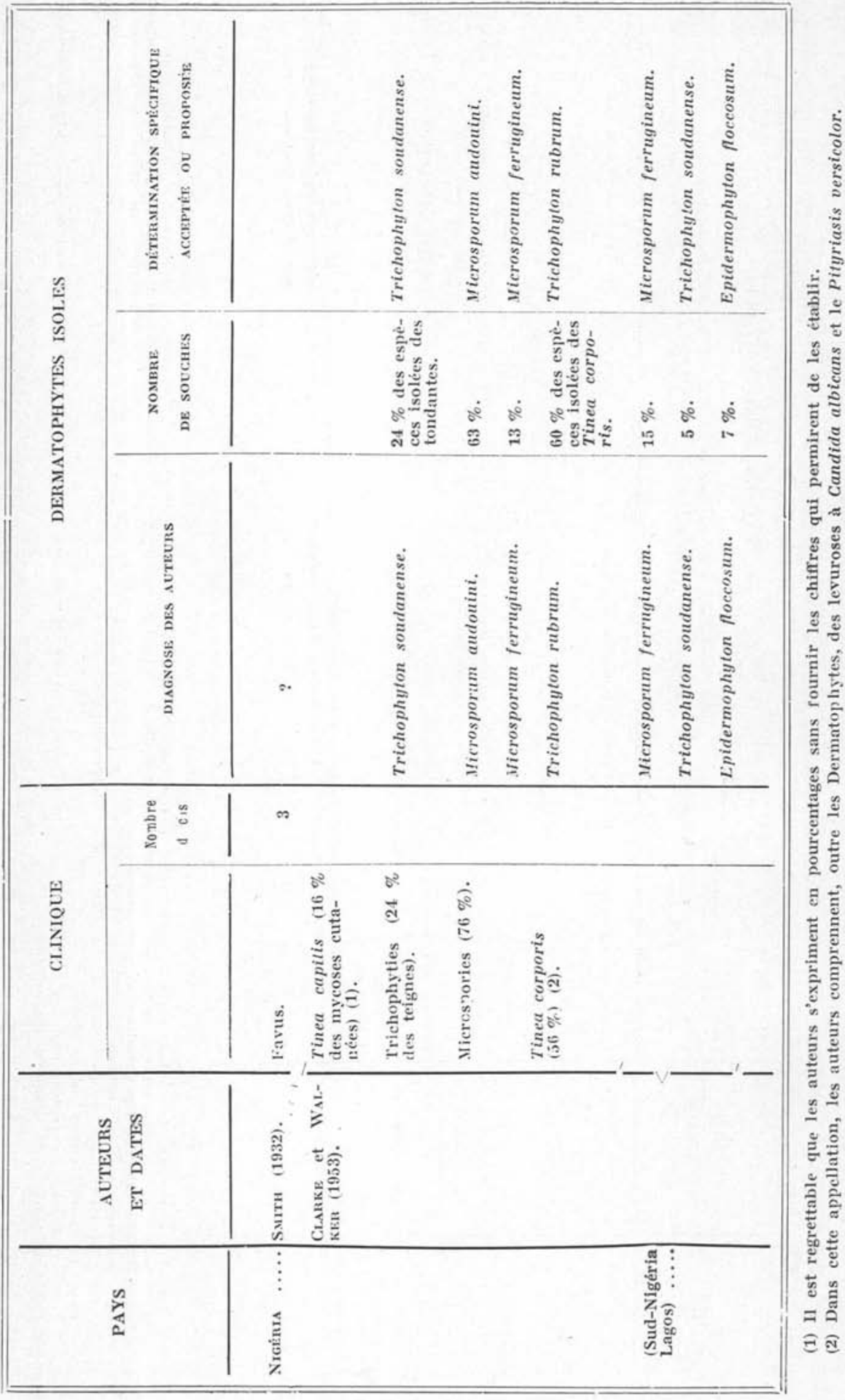




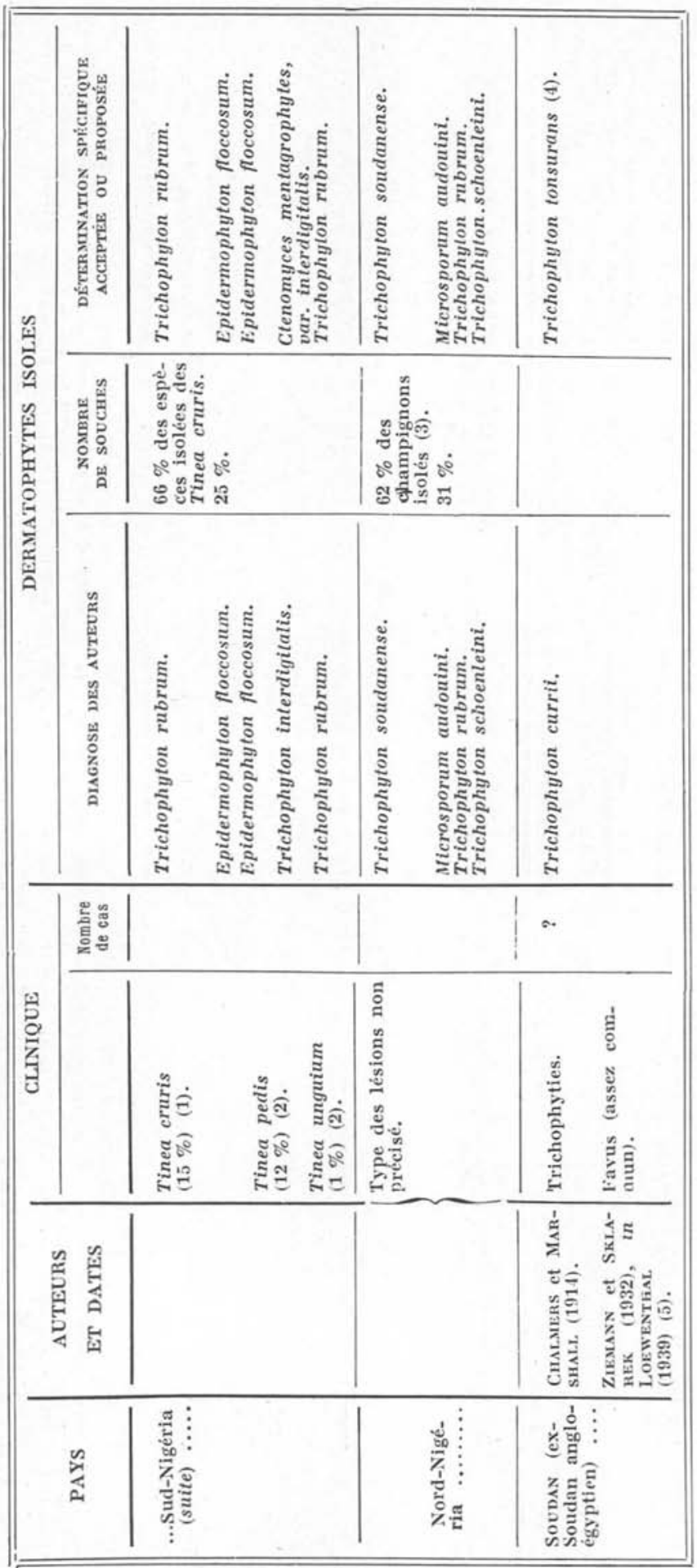

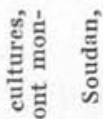

跣

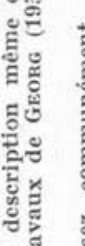

离

ฮै

跣 导

苟 总

:

ค

व

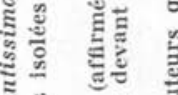

हू

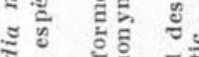

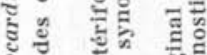

¿ 饲

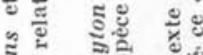

ปू. ส

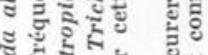

ฐึّ

‥ II \&

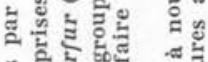

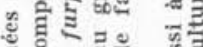

워웛

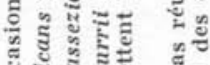

ชี ปี

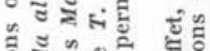

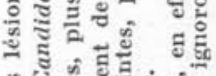

क्

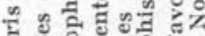

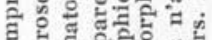

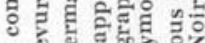

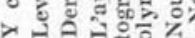

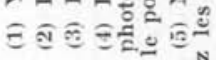

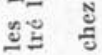

Ann. de Parasitologie, T. XXXIII, No 5-6, 1959. 


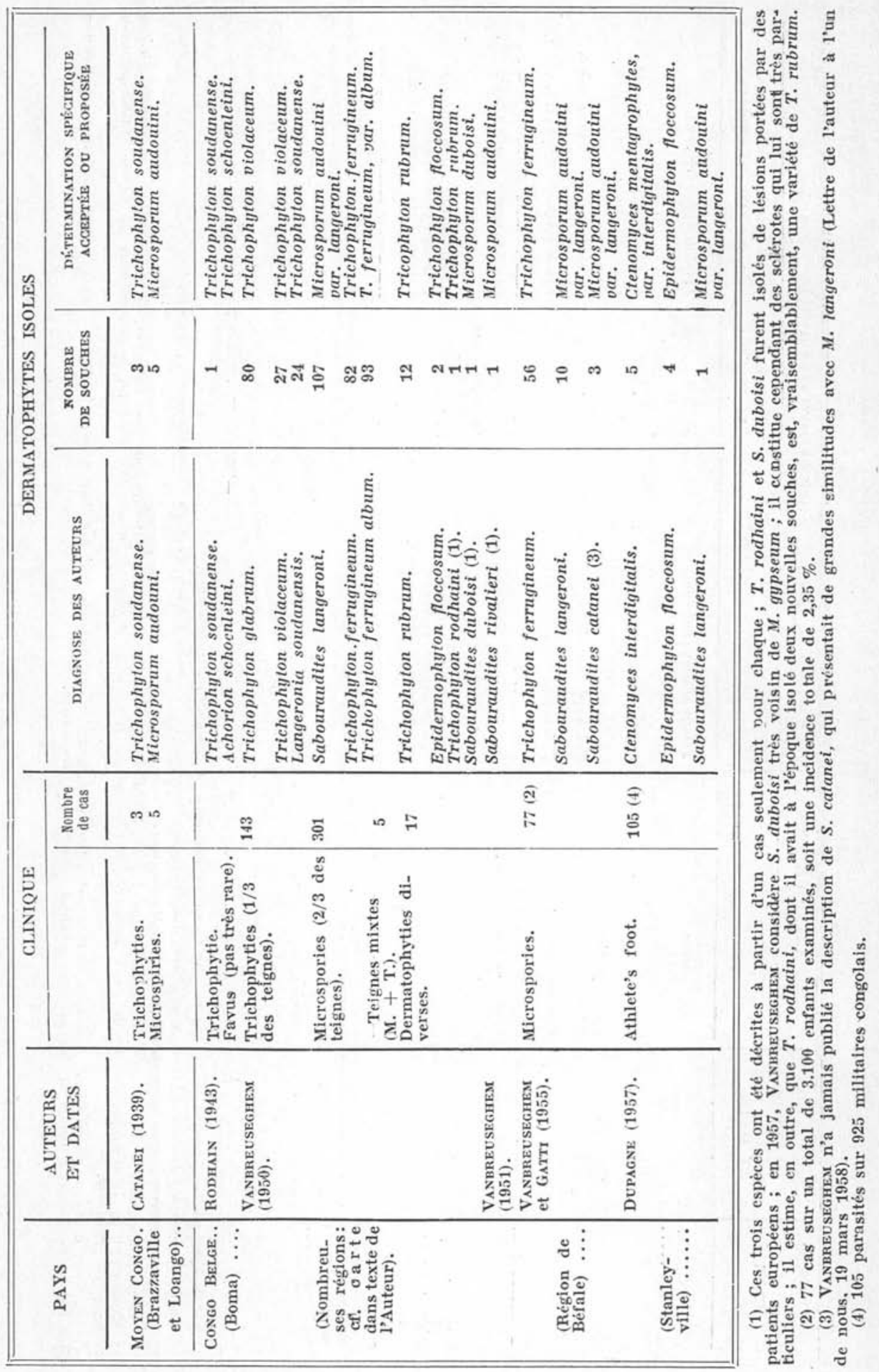




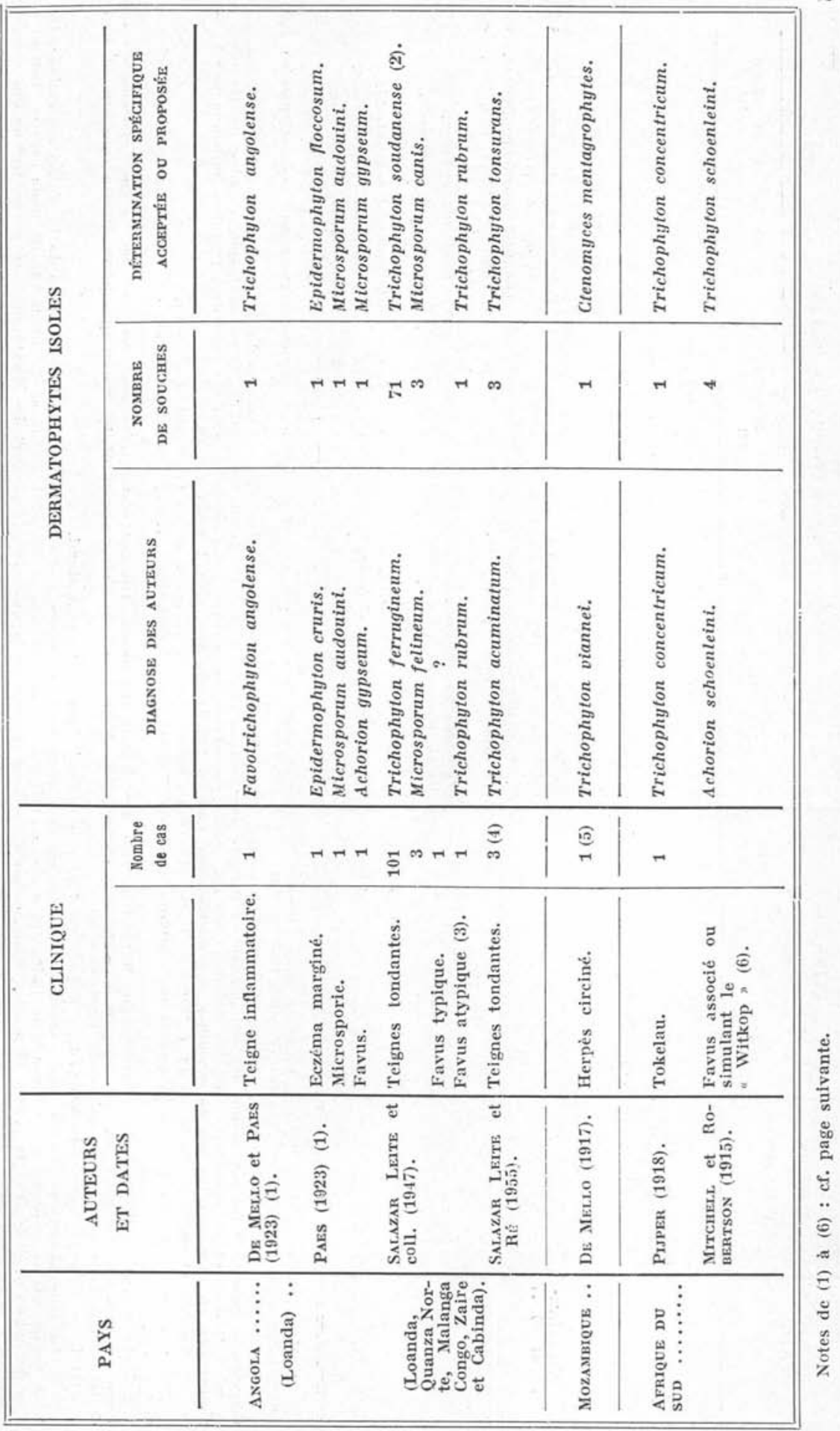




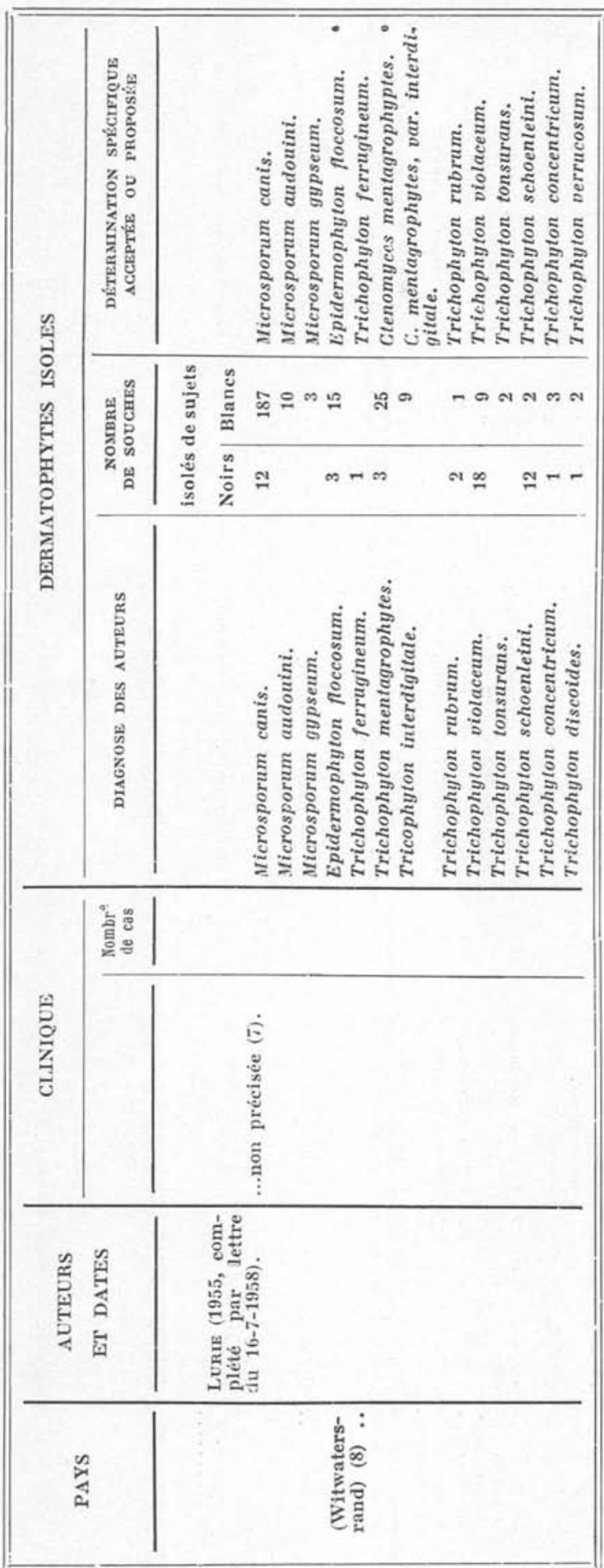

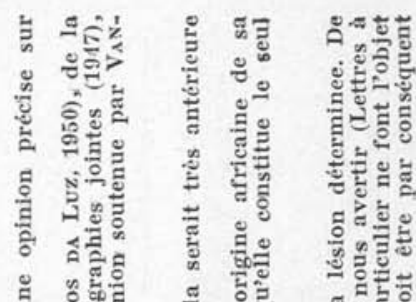

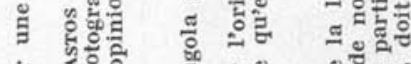

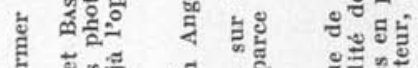

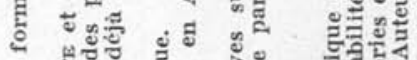

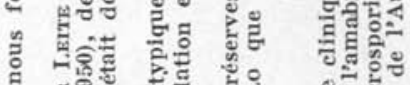

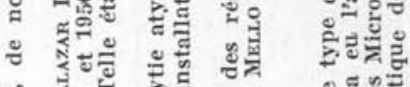

ث

ड

E

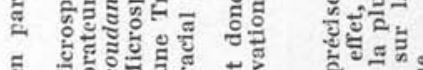

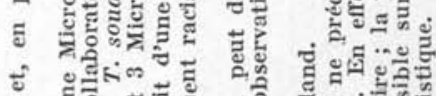

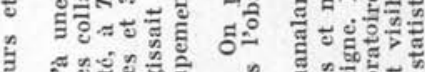

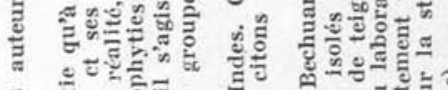

c

-

E

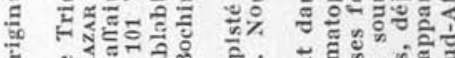

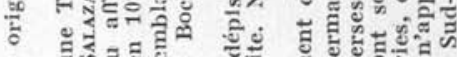

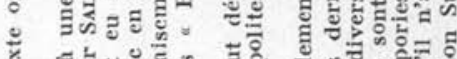

๘

-

ัरें

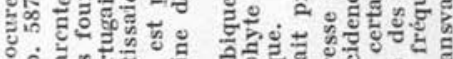

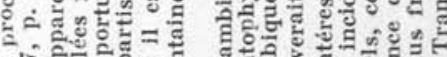

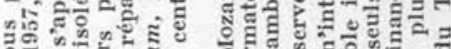

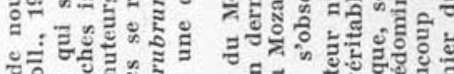

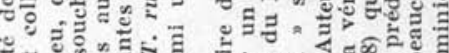

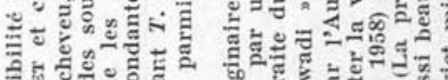

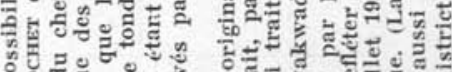

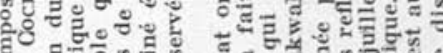

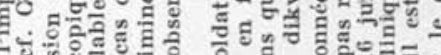

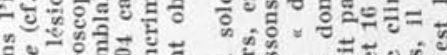

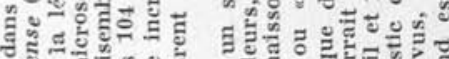
ฐั0 क्ष्ठ ๓ สี

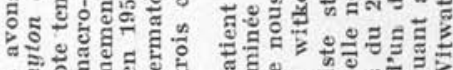
แ

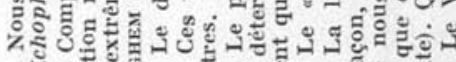
อง จ 论 $=$ 팸 
lacune de notre bibliographie, aucune recherche n'a été réalisée dans un vaste ensemble de territoires situés à l'Est du Congo Belge et de l'Angola. Aussi, nos commentaires n'auront-ils d'autre prétention que de faire le point très provisoire du modeste acquis actuel et d'attirer l'attention des auteurs sur divers problèmes cliniques, épidémiologiques ou mycologiques, dont il est indispensable de prendre conscience pour rendre au maximum efficientes les recherches ultérieures sur les dermatophyties et les dermatophytes d'Afrique.

\section{A) Considérations sur la répartition des teignes} et de leur forme clinique en Afrique Noire

Ce sont naturellement les teignes qui retiendront presque toute notre attention parce que, dermatophyties majeures, elles ont fait l'objet de la plupart des enquêtes effectuées; elles représentent par conséquent l'aspect le moins mal connu des Dermatophyties africaines. Il est, en effet, encore beaucoup trop tôt pour essayer de dégager les idées générales concernant les lésions cutanées de la peau glabre (non concomitantes d'une teigne), et nous ne ferons qu'une allusion au Tokelau qui existerait peut-être très exceptionnellement en Afrique, où il a été signalé cinq fois en Afrique du Sud (dont la population d'ailleurs très hétérogène comprend certains éléments d'origine asiatique) et en Libéria (Poindexter) (5).

(5) Le rapport de Poindexter, écrit en 1947 à la suite d'une mission de 18 mois cffectuée à l'instigation du Service de la Santé Publique des Etats-Unis, apporte quelques renseignements sur les mycoses du Libéria. Malheureusement, la partie de la publication qui traite de ce sujet est relativement mince, et il semble que l'auteur ne soit pas spécialisé en la matière. Nous relevons en effet, dans un texte imprécis que dans une des aires de prospection, sur 226 personnes examinées, $15 \%$ présentaient une maladie fongique, teigne ou levurose. A la fin de son travail, il précise encore: « The six most prevalent types of fungus infection in 665 confirmed cases occured in the following order of relative prevalence : Tinea versicolor ; $T$. imbricata ; $T$. circinata; $T$. capitis, an Achorion or monilia like organism and $T$. unguium $\gg$. En ce qui concerne les Dermatophytes, il signale les espèces suivantes: Trichophyton gypseum, Trichophyton purpureum, Trichophyton floccosum et Trichophyton concentricum. On cst évidemment surpris de voir le Tokelau, maladie essentiellement localisée à l'Insulinde et à l'Amérique du Sud et du Centre, plus commun au Libéria que l'Herpès circiné ou la teigne du cuir chevelu, et de constater que la liste des cspèces mycologiques cultivées ne renferme aucun des Dermatophytes agents babituels de tondante. Aussi, ne faut-il accepter, à notre avis, qu'avec de très prudentes réserves les diagnosties et les diagnoses proposés par Poindexter. 


\section{$1^{\circ}$ Le Favus}

Près de vingt ans après les travaux de Catanéi $(1939, a, b, c)$, il se confirme que le Favus, si fréquent dans le Nord de l'Afrique (6), est généralement rare dans les territoires de peuplement noir (7). Son nom cependant se trouve cité dans les publications qui traitent des teignes du Soudan Français, du Nigéria, de l'Angola, du Congo Belge, du Libéria (8), du Soudan (ex-Soudan Anglo-Egyptien) et de l'Afrique du Sud. Mais nous avons déjà posé des réserves sur le cas issu du Soudan Français (9). Des trois cas qui furent signalés en Angola (Paès, 1923 ; Salazar Leite et coll., 1947), le premier a pour agent Microsporum gypseum et, le second, Trichophyton rubrum ; quant au troísième le seul cliniquement typique, il n'a pas donné lieu à une culture. En ce qui concerne l'ancien Soudan Anglo-Egyptien, la proximité de l'Egypte, où le Favus est commun comme partout en Afrique du Nord, y rend très vraisemblable la présence de cette teigne. Par ailleurs, si on acceptait la proposition de Clarke et Walker (1953), qui pensent le Favus lié à des facteurs climatiques, on pourrait s'expliquer sa découverte en Afrique du Sud, où il serait très commun au moins dans les territoires utilisés comme réserves de natifs (Lurie, 1955), et dans la partie du NordNigéria, où il serait aussi assez fréquent (Clarke et Walker, 1953). En effet, on trouve dans l'extrême Sud Africain un climat de type méditerranéen et un peu plus au Nord, dans le Sud-Ouest, des régions de climat désertique ; ce même climat désertique s'observe dans le Nord du Nigéria (dans le Sud du pays qui est humide, le Favus fait défaut). Mais une réserve s'impose sur l'interdépendance du Favus et des climats chauds et secs ; il ne faut pas oublier en effet que cette teigne n'est pas rare dans de nombreux pays de climats tempérés, assez froids et parfois notablement humides, comme le Nord de la France par exemple (Huriez et coll., 1956).

(6) Sauf apparemment en Tripolitaine (on trouvera la bibliographie et une revue générale des teignes du Nord de l'Afrique dans le travail de Coutelen et coll. concernant la Tunisie, et publié en 1956).

(7) Il est possible d'ailleurs qu'un facteur racial intervienne dans cette relative rareté du Favus chez les Noirs; les particularités de structure et de composition de leurs cheveux, par exemple, ne sont peut-être pas favorables à l'évolution du Trichophyton schoenleini. Hazen (1914-1935), aux Etats-Unis, n'a pas trouvé un seul Favus à l'examen de 11.729 personnes de couleur, et Catanei (1933), dans les oasis du Sud-Oranais, constatait que les négroïdes sont presque exclusivement atteints de Trichophytie et très rarement de Favus. Mais il est évident qu'il est encore beaucoup trop tôt pour être affirmatif sur l'influence de ce facteur racial.

(8) Cf. note (1) page précédente.

(9) Cf. note (4) du Tableau II A intéressant le Soudan Français. 
A coup sùr, on serait plus facilement convaincu de la présence du Favus dans l'ensemble des territoires africains cités plus haut si les divers auteurs avaient fourni, ou eu la possibilité de fournir, de plus amples descriptions cliniques, précisé le type des lésions macro- et microscopiques du cheveu malade, et, dans une discussion critique concernant le champignon isolé, éliminé la possibilité d'une confusion éventuelle de Trichophyton schoenleini, agent cosmopolite du Favus, avec d'autres dermatophytes. Nous ne pouvons en effet que partager l'opinion de Vanbreuseghem (1957, et lettre du 19 mars 1958 à l'un de nous) sur la cause d'erreur que peut entrainer la ressemblance de certaines cultures faviformes et apigmentées ( $T$. ferrugineum, $T$. soudanense) avec l'agent classique du Favus (10). On sait que cet auteur, en dépit d'une vaste expérience des teignes congolaises (11), n'a jamais eu l'occasion de confirmer la présence du Favus au Congo Belge où Rodhain l'avait signalé antérieurement (1943) (12). Il est bon de préciser d'ailleurs qu'il s'agissait souvent, comme en Afrique du Sud (Mitchell et Robertson, 1924), de cas atypiques où la tête était casquée de croûtes blanches, et que, peut-être, Rodhain avait eu affaire en réalité à des lésions cutanées entrainées par des carences alimentaires, éventuellement compliquées par une Trichophytie (cf. p. 10). Notons cependant que cet auteur précise avoir observé dans certains cas des godets qui avaient envahi les épaules et la poitrine des malades.

En définitive, on voit que la démonstration de l'existence du Favus classique à Trichophyton schoenleini soulève des difficultés en Afrique Noire et qu'il faudra par conséquent attendre des recherches ultérieures, effectuées avec la conscience de ces difficultés, avant de pouvoir discuter valablement la répartition et la fréquence de cette teigne dans les divers terriioires africains.

\section{$2^{\circ}$ LES TONDANTES MICROSPORIQUES ET TRICHOPHYTIQUES}

Des commentaires sur le Favus qui précèdent, il ressort que les tondantes représentent, en fait, la quasi-totalité des teignes de l'Afrique Noire. Autre différence capitale avec l'endémie teigneuse

(10) Cf. 1a variabilité de T. ferrugineum (Vanbreuseghem, 1950) et les colonies de T. soudanense simulant T. schoenleini (Cochet et coll., 1957).

(11) Dans son article de 1957 , il précise qu'à l'examen de 15.000 indigènes du Congo Belge et du Ruanda-Urundi il n'a trouvé aucun cas de Favus.

(12) Rodhain a publié en 1943 le résultat d'observations effectuées sur des enfants de 6 à 15 ans, vingt ans auparavant (1924-1925) dans le Mayumbé, à Boma, à l'embouchure du Congo. T. schoenleini (= Achorion schoenleini) y aurait été isolé plusieurs fois. Au dire des malades, certains patients guériraient spontanémeni vers l'âge de la puberté, et l'auteur a pu constater lui-même chez des adultes l'existence d'alopécies cicatricielles dues très vraisemblablement à des Favus guéris. 
du Nord de l'Afrique, les Microspories sont ici autochtones ; et, loin d'être rares, elles peuvent l'emporter en fréquence sur les Trichophyties. Voici à ce sujet quelques précisions supplémentaires :

$1^{\circ}$ La fréquence des tondantes, quoique très variable suivant le territoire et à l'intérieur d'un territoire selon la région considérée, paraît souvent assez élevée et n'est sans doute nulle part négligeable. Malheureusement, peu de statistiques à notre connaissance apportent des précisions à ce sujet; on peut citer toutefois celles de Cochet et coll. (1957) au Cameroun où, à Yaoundé et dans un rayon d'une centaine de kilomètres, un pourcentage de 32,55 porteurs a été trouvé parmi 2.660 enfants scolarisés. Au Congo, Vanbreuseghem a fourni d'assez nombreuses statistiques régionales qui illustrent parfaitement la diversité locale de la diffusion des teignes tondantes : dans le territoire de Béfalé, choisi avec Gatti (1955) pour tenter de déterminer l'incidence naturelle des teignes infantiles, en dehors de toute influence civilisatrice, il a examiné 3.100 enfants, dont $2,35 \%$ seulement étaient parasités. Puis, en 1957 , il a montré que, si la fréquence des tondantes est relativement faible à Luisa $(2,9 \%)$, à Opala (3\%), à Stanleyville $(5,9 \%)$, à Bengamisa $(6,3 \%)$ et à Léopoldville $(6,7 \%)$, elle s'élève déjà à Elisabethville (15\%), à Usumbura $(15,7 \%)$ et à Bukavu $(20 \%)$, pour atteindre des pourcentages plus importants encore dans les montagnes de Ruanda-Urundi $(32,5 \%)$, dans celles du Sud de Bukavu (25,5 à $37 \%)$ et à Luluabourg (40\%).

$2^{\circ}$ En 1957, Vanbreuseghem a tenté d'analyser les facteurs susceptibles d'expliquer la variabilité des pourcentages précédemment cités. Après avoir considéré l'influence éventuelle de la virulence des souches, de l'évolution sociale et économique et de la densité des populations, il a attaché un intérêt tout particulier au régime alimentaire. Constatant, en effet, que les taux d'infestation élevés frappent les populations dont le régime alimentaire est plus particulièrement carencé en protéines, il a émis l'hypothèse qu'une telle carence rend le cheveu malade et que sa résistance mécanique à l'invasion du champignon se trouve diminuée, ce qui favorise le développement du dermatophyte.

Il est bien difficile, en réalité, compte tenu de l'intrication et de la dépendance des divers facteurs cités, de préciser leur intérêt respectif dans la diffusion des tondantes. Une pareille tentative nécessiterait à tout le moins des statistiques nombreuses et importantes qui permettraient une sévère étude critique. Ajoutons encore que, - maladies contagieuses et avant tout maladies de l'enfance -, leur fréquence doit être à notre avis fortement influencée par 
la scolarisation qui n'exprime d'ailleurs qu'un aspect particulier de la densité de la population. Nous pensons aussi qu'indépendamment du terrain, favorable à des degrés divers à la multiplication des teignes, que peut présenter une population (plus ou moins dense, plus ou moins soucieuse de l'hygiène, plus ou moins carencée, etc...), interviennent des facteurs encore mystérieux, semblables à ceux qui, en France, dans des groupes scolaires très comparables par ailleurs, entretiennent un état endémique des plus discrets ou, au contraire, déterminent une poussée épidémique parfois spectaculaire.

$3^{\circ}$ Ce n'est pas seulement la fréquence absolue de la teigne tondante qui change avec les pays, mais encore la fréquence relative de ses deux formes cliniques, Microsporie et Trichophytie. Le tableau III fait le point de l'état actuel de nos informations pour quelques territoires; mais il est certain que les chiffres que nous possédons actuellement sont généralement beaucoup trop faibles pour être significatifs. Cependant, la Trichophytie semble prédominer au Cameroun, en Angola, au Soudan Français, en Guinée Française, dans le Nord du Nigéria ; par contre, la Microsporie l'emporte au Congo belge, dans le Sud du Nigéria et en Afrique du Sud.

Mais, en réalité, à l'intérieur même de chacun de ces territoires, la fréquence relative des Microspories et des Trichophyties peut aussi différer considérablement suivant la région considérée. Le Nigéria nous en a fourni un bon exemple et il en va de même en Guinée où la Trichophytie serait nettement plus rare sur la côte (Joyeux, 1912) ; le Congo, enfin, nous administre encore une excellente démonstration de ce fait: dans la région de BanzyvilleYakoma, à la frontière de l'Oubangui-Chari, à Stanleyville (Vanbreuseghem, 1950), dans la région de Béfalé (Vanbreuseghem et Gatti, 1955), les Microspories semblent exister seules ; à Luluabourg et à Elisabethville (Vanbreuseghem, 1957), les deux formes de tondantes s'équilibrent sensiblement, les Microspories restant toutefois prédominantes ; enfin, à Usumbura, elles ne représentent plus que $10 \%$ des tondantes avant de disparaitre totalement dans les montagnes de Ruanda-Urundi (Vanbreuseghem, 1957).

$\mathrm{Si}$ nous éprouvons des difficultés à envisager une explication plausible de la diffusion géographique variable de la teigne tondante, il est bien évident que nous sommes a fortiori incapables de suggérer la moindre hypothèse susceptible d'apporter quelque éclaircissement sur la distribution éventuellement si changeante de ses deux formes, microsporique ou trichophytique, suivant le lieu considéré. 
TABLEAU III. - Fréquence relative

des microspories et des trichophyties dans quelques territoires

\begin{tabular}{|c|c|c|c|c|c|}
\hline \multirow{2}{*}{ TERRITOIRES } & \multirow{2}{*}{$\begin{array}{l}\text { Auteurs } \\
\text { ET DATES }\end{array}$} & \multicolumn{2}{|c|}{ MICRospories } & \multicolumn{2}{|c|}{ TRICHOPHYTIES } \\
\hline & & $\begin{array}{l}\text { Nombre } \\
\text { de cas }\end{array}$ & $\%$ & $\begin{array}{l}\text { Nombre } \\
\text { de cas }\end{array}$ & $\%$ \\
\hline Sénégal $\ldots \ldots \ldots$. & $\begin{array}{l}\text { Courmont (1896) } \\
+ \text { Biguet et coll. } \\
\text { non publié. }\end{array}$ & 7 & & 7 & \\
\hline Soudan français. & Catanéi (1939 a). & 1 & & 13 (*) & \\
\hline Guinée française. & $\begin{array}{l}\text { Joyeux }(1912)+ \\
\text { Catanéi }(1939 a) \text {. }\end{array}$ & 5 & & $36(*)$ & \\
\hline Haute-Volta... & $\begin{array}{l}\begin{array}{l}\text { Biguet et coll. } \\
(1958)(*) .\end{array}\end{array}$ & 21 & 12,3 & 148 & 86,6 \\
\hline Dahomey ...... & Catanéi $(1939 a)$. & 6 & & 4 & \\
\hline $\begin{array}{l}\text { Togo } . . . . \ldots \ldots \\
\text { Nigéria : }\end{array}$ & Catanéi $(1939 a)$. & 14 & & 4 & \\
\hline $\begin{array}{l}\text { Nigeria : } \\
\qquad \begin{array}{l}\text { Sud } \ldots \ldots \ldots \ldots \\
\text { Nord } \ldots \ldots \ldots \ldots\end{array}\end{array}$ & $\begin{array}{l}\text { Clarke et Walker } \\
\text { (1953). }\end{array}$ & & $\begin{array}{l}76 \\
31\end{array}$ & & $\begin{array}{l}21 \\
62\end{array}$ \\
\hline Cameroun $\ldots$. . & $\begin{array}{l}\text { Cochet et coll. } \\
(1957)(*) \text {. }\end{array}$ & 156 & 20 & 707 & 80 \\
\hline Congo belge .... & $\begin{array}{l}\text { Vanbreuseghem } \\
(1950)\left({ }^{*}\right) .\end{array}$ & 301 & 66 & 143 & 34 \\
\hline Angola $\ldots \ldots \ldots$ & $\begin{array}{l}\text { Salazar Leite et } \\
\text { coll. (1947 et } \\
\text { 1955). }\end{array}$ & 3 & & 104 & \\
\hline Afrique du Sud.. & $\begin{array}{l}\text { Lurie (lettre du } \\
16-7-1958) .\end{array}$ & $209(* *)$ & 88,5 & $27(* *)$ & 11,5 \\
\hline
\end{tabular}

(*) Cas de teigne mixte non compris.

(**) Les cas observés chez les sujets de race blanche et noire sont additionnés.

$4^{\circ}$ En ce qui concerne l'aspect clinique des teignes africaines, il convient de noter d'abord qu'il est dans l'ensemble, en dépit de quelques précisions apportées par Catanéi $(1939 c)$, assez mal connu, même au Congo Belge (Vanbreuseghem, 1955) (13). Il est vraisemblable d'ailleurs que la lésion doit bien souvent être remaniée, entre autres par l'infection surajoutée, la pédiculose et occasionnellement par l'usage de cosmétiques.

(13) A cette date, Vanbreuseghem, dans un travail intitulé : « Le Congo Belge et la mycologie médicale », écrivait: "Si nous savons qu'il y a au Congo Belge des dermatophytes du cuir chevelu et des dermatophytes de la peau glabre, nous ignorons leur aspect et leur évolution ». Il avait précisé toutefois en 1950 que l'aspect clinique des lésions trichophytiques et microsporiques paraissait classique et que la présence de lésions cutanées concomitantes était un argument solide en faveur de la Microsporie. 
Il existe en outre deux problèmes au moins, soulevés par les tondantes africaines, qui nous paraissent mériter une attention particulière de la part des auteurs qui ont la possibilité de les étudier sur place :

a) Les teignes mixtes, à la fois microsporiques et trichophytiques. Elles ne doivent pas être très rares en Afrique Noire où leur présence a été signalée en Guinée Française, au Congo, au Cameroun, au Nigéria et en Haute-Volta. Jusqu'ici, cependant, elles ne semblent avoir été décelées que par les cultures. Il conviendrait de préciser, outre leur fréquence réelle, leur aspect clinique et, en particulier, la part relative prise par chaque tondante dans les lésions.

b) Les trichophyties de l'adulte. Dès 1919, Salanoue-Ipin les affirmait fréquentes. Dans les montagnes de Ruanda-Urundi, $10 \%$ des montagnards sont parasités d'après Vanbreuseghem (1957). Il serait intéressant de rechercher systématiquement cette infestation des adultes et de comparer sa fréquence à celle des enfants (14).

\section{B) Discussion sur les espèces de Dermatophytes isolées et leur répartition géographique}

Avant d'aborder cette discussion, précisons que nous reconnaissons chez les Dermatophytes les distinctions génériques suivantes :

- Microsporum Gruby, 1843 :

Il nous parait inutile d'abandonner ce vieux nom de genre pour Sabouraudites Ota et Langeron, 1923.

- Trichophyton Malmsten, 1845 :

Bien que nous ayons souhaité antérieurement le maintien au moins provisoire du genre Achorion Lebert, 1843, nous jugeons préférable à la réflexion d'y renoncer, suivant en cela l'exemple de la quasi-totalité des mycologues ; quant au genre Langeronia Vanbreuseghem, 1950, son individualisation nous paraît très insuffisamment établie, et nous le considérons comme synonyme de Trichophyton.

- Ctenomyces Eidam, 1880 :

Nous acceptons de ranger dans ce genre les anciens Trichophyton microïdes de Sabouraud.

- Epidermophyton Lang, 1879.

(14) A Marseille, Ranque et Temime (1957) ont fait une statistique sur des sujets Nord-Africains et trouvé les pourcentage d'infestation suivants : $8,7 \%$ chez les enfants (9.444 examinés) et $0,4 \%$ chez les adultes ( 8.900 examinés). 
En ce qui concerne les espèces valides, nous partageons généralement les conceptions américaines issues en particulier des recherches d'Emmons, de Georg et de Conant et qui ont succédé à un très important travail publié en 1934 par le premier auteur cité. Toutefois, lorsque les anciennes espèces tombées en synonymie étaient bien décrites et, de ce fait, parfaitement reconnaissables, il nous semble préférable, afin d'être plus précis et de ménager l'avenir, de les maintenir en tant que variétés ou que formes culturales à l'intérieur de l'espèce «collective » (par exemple, Ctenomyces mentagrophytes, variété granulosum ou variété interdigitale).

Compte tenu des remarques précédentes et des synonymies généralement acceptées, nous avons, dans le tableau II, modifié comme suit certaines appellations des auteurs cités : le genre Sabouraudites est mis en synonymie avec Microsporum, les genres Langeronia et Favotrichophyton avec Trichophyton; Microsporum felineus correspond à M. canis; Achorion schoenleini devient Trichophyton schoenleini, Achorion gypseum est appelé Trichophyton gypseum et $T$. circonvolutum est rapporté à $T$. tonsurans ; Microsporum ferrugineum, pour sa part, est transporté dans le genre Trichophyton, tandis que Trichophyton mentagrophytes passe dans le genre Ctenomyces; nous considérons Trichophyton viannei comme l'un des multiples aspects culturaux de Ctenomyces mentagrophytes; Epidermophyton cruris et Trichophyton discoides deviennent respectivement $E$. floccosum et $T$. verrucosum (15).

Ce premier travail effectué, nous restons en présence de nombreuses espèces dont l'importance, il est vrai, est très inégale. Il y a peu de commentaires à faire sur les espèces des deux genres monotypes Ctenomyces et Epidermophyton: C. mentagrophytes et E. floccosum sont cosmopolites; leur découverte se multipliera, selon toute vraisemblance, au fur et à mesure que les auteurs s'intéresseront à l'étude des dermatophyties des téguments dits " glabres » et plus spécialement aux lésions d'Athlete's foot. Par contre, les espèces des genres Trichophyton et Microsporum méritent de retenir plus longtemps notre attention.

\section{1) Considérations sur LeS espèces dU GENRE Trichophyton}

Nous n’insisterons pas sur T. schoenleini. Nous avons déjà été amenés à évoquer les problèmes intéressant ce dermatophyte en traitant du Favus et nous avons mis l'accent, après Vanbreuseghem

(15) Nous avons modifié certaines autres diagnoses des auteurs; on lira la brève justification de ces modifications dans les notes du Tableau II. 
(1957), sur les confusions que peut entrainer le polymorphisme de certains Trichophyton.

Quant à $T$. concentricum, agent du Tokelau, son importance est assez mince, car il y a peu de chance que des recherches ultérieures confirment le travail de Poindexter au Libéria, et il n'a été, par ailleurs, isolé que cinq fois en Afrique du Sud.

$T$. ferrugineum est une espèce d'un intérêt capital, qu'on a jusqu'ici signalée parmi les agents des Microspories au Congo Belge et au Cameroun où son rôle est prédominant, au Nigéria où il représente encore $13 \%$ des dermatophytes isolés dans les Microspories et en Haute-Volta où il semble beaucoup moins fréquent. Il est vraisemblable qu'il sera retrouvé dans la plupart des pays africains qui avoisinent les précédents lorsque les investigations auront été plus poussées. Il parait, par contre, être rare en Afrique du Sud où il n'a été isolé qu'une seule fois de plus de 200 Microspories (lettre de Lurie du 16 juillet 1958) ; cependant, la statistique de Lurie concernant les dermatophytes intéresse les Blanes (274) beaucoup plus que les Noirs (54), et des recherches ultérieures peuvent considérablement modifier l'impression qui se dégage actuellement des travaux de cet auteur.

T. rubrum est une espèce cosmopolite responsable de nombreuses lésions cutanées et on peut répéter à son propos ce qui a été dit plus haut pour $C$. mentagrophytes et E. floccosum. Au Nigéria, cette espèce a été mise en évidence dans 60 à $66 \%$ des cas de dermatophyties de la peau " glabre 》(Clarke et Walker, 1953) (16). Elle n'est pas rare au Congo Belge et elle existe au Cameroun, en Angola et en Afrique du Sud. Elle détermine parfois des tondantes trichophytiques (Haute-Volta, Angola). De l'avis personnel de Vanbreuseghem, en 1957, il est probable qu'il convient de rattacher à cette espèce extrêmement polymorphe la souche atypique qu'il décrivit en 1949 (17) sous le nom de $T$. rodhaini et qu'il eut ensuite l'occasion d'isoler plusieurs fois.

A l'origine de certaines tondantes trichophytiques, sous des formes culturales variées et généralement plus vivaces que les souches européennes, on rencontre Trichophyton tonsurans dans plusieurs territoires et, sous réserve que les diagnoses des auteurs soient

(16) Deux formes culturales apparemment stables sont isolées par Clarke et Walker; la plus fréquemment rencontrée est cratériforme, poudreuse et riche en macroconidies; la seconde est cotonneuse, forme de nombreuses microconidies, mais seulement occasionnellement des macroconidies. C'est à ce type de culture que se rapportaient les deux souches que nous avons isolées de Haute-Volta.

(17) On retrouvera sa description in Vanbreuseghem, 1950. 
exactes, on peut supposer cette espèce présente partout. Cependant, les cas d'infestation semblent jusqu'ici assez rares. Sans qu'on ne puisse rien affirmer, il est vraisemblable que ces souches soient autochtones (sauf en Afrique du Sud où les deux sujets parasités sont des Blancs). Il est possible d'ailleurs que des enquêtes ultérieures dans de nouveaux territoires montrent que, dans certains d'entre eux, T. tonsurans est beaucoup plus fréquent (18).

Quant à $T$. violaceum, agent presque exclusif des Trichophyties du Nord de l'Afrique, il est beaucoup moins répandu en Afrique Noire. Vanbreuseghem (1950), cependant, l'a cultivé de nombreuses fois au Congo Belge où, avec sa variété blanche, il est à l'origine de $80 \%$ des Trichophyties. Il existe aussi en Afrique du Sud où il parasite des sujets de race blanche et de race noire. Il est concurrencé partout ailleurs par diverses espèces : T. yaoundei est le dermatophyte le plus fréquemment isolé au Cameroun. T. gourvili existe au Sénégal, au Soudan Français, en Haute-Volta et au Togo, territoires groupés au nord-ouest de l'Afrique Noire. Sa fréquence en Haute-Volta est particulièrement notable puisque nous l'avons isolé trois fois plus souvent que $T$. soudanense. Cette dernière espèce occupe d'ailleurs une place toute particulière dans l'étiologie des Trichophyties. Il est en effet le plus largement répandu des Trichophyton d'Afrique Noire. Il ne parait faire défaut qu'en Afrique du Sud et au Soudan (ce dernier territoire étant en réalité bien peu étudié). Il constitue parfois la seule espèce isolée des lésions trichophytiques, comme en Guinée Française et en Angola (à trois souches de T. tonsurans près pour l'Angola) (19).

Le tableau IV et la carte A $\left(19^{\text {bis }}\right)$ illustrent, telles qu'on peut se les représenter actuellement, la répartition géographique et l'importance relative des principaux Trichophyton en Afrique Noire (la carte A est limitée aux principales espèces qui déterminent des Trichophyties).

(18) Des différences considérables dans la fréquence de ce dermatophyte ont. en effet, déjà été notées dans le nord de l'Afrique. C'est ainsi qu'en Tripolitaine, $T$. tonsurans a été isolé 27 fois et $T$. violaceum 59 fois (Lapidus, 1950), alors qu'en Tunisie, par exemple, $T$. tonsurans est beaucoup plus rare, puisque 17 souches seulement furent observẻes contre 1.058 souches de $T$. violaceum (Coutelen et coll., 1956).

(19) Cf. note 2 du Tableau II E concernant la diagnose en Angola des souches étiquetées $T$. ferrugineum par Salazar Leite et coll. (1947).

$\left(19^{\mathrm{b}+\mathrm{s}}\right)$ C'est par erreur que Trichophyton gourvili est figuré sur cette carte comme étant connu en Guinée. 


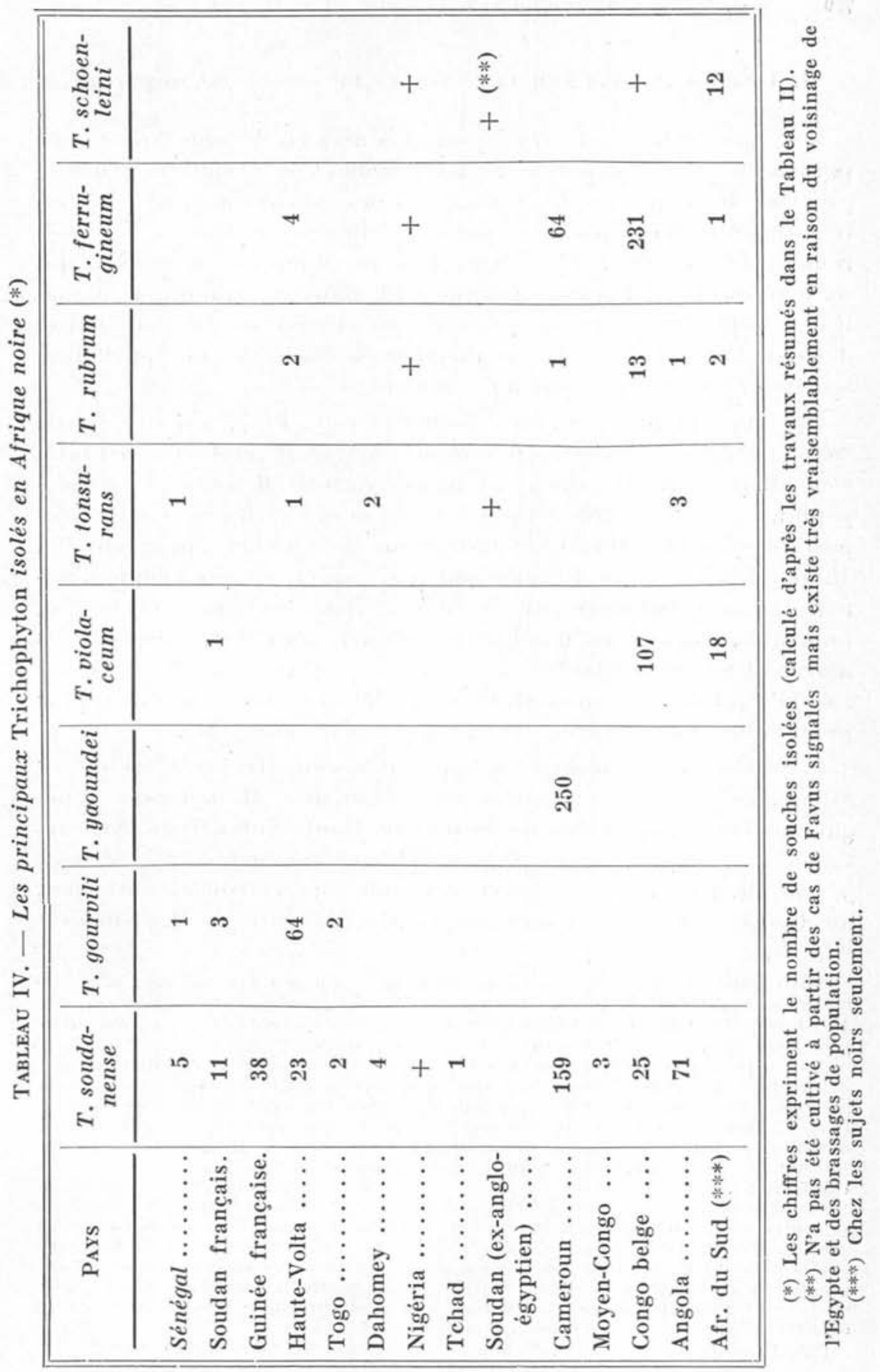




\section{2) Considérations SUR LeS espèces du GenRe Microsporum}

Les espèces du genre Microsporum isolées en Afrique Noire nous paraissent poser un problème assez ardu. Une première difficulté provient de ce que les déterminations des auteurs ne sont pas systématiquement comparables parce qu'elles se ressentent nécessairement de facteurs subjectifs et de conceptions taxonomiques qui ne nous sont pas toujours connues. Et, dans ces conditions, l'analyse critique, fréquemment malaisée en matière de dermatophytes, devient impossible lorsque la diagnose se limite à une appellation spécifique que n'étaye pas un commentaire.

Quelques-uns d'entre nous (Cochet et coll., 1957) ont suggéré de rassembler les Microsporum sous le binôme $M$. audouini, à l'intérieur duquel subsisteraient en tant que variétés $M$. audouini anthropophile et $M$. canis (20) zoophile. Ils avaient été incités à cette proposition par l'isolement au Cameroun de souches apparentées à $M$. audouini, mais en différant par leur aspect macroscopique d'ailleurs assez variable et par l'existence, dans certaines souches au moins, de fuseaux en nombre inhabituel pour cette espèce. Ils n'avaient guère été tentés de créer une espèce nouvelle pour ces souches qui semblaient se situer entre $M$. audouini et $M$. canis, plus proches cependant à leur avis de la première citée (21).

Considérons maintenant les faits publiés en Afrique Noire où les espèces suivantes ont été citées par les auteurs : M. audouini (Sénégal, Soudan Français, Guinée Française, Haute-Volta, Togo, Nigéria, Cameroun, Moyen-Congo, Angola, Afrique du Sud) (22) ; M. langeroni, $M$. rivalieri, $M$. catanei (ces trois espèces isolées seulement au Congo Belge par Vanbreuseghem); M. canis (=M. felineum)

(20) Cochet et coll. (1957) ajoutaient $M$. gypseum. En fait, ce dermatophyte est bien caractérisé; il ne semble pas exister de souches intermédiaires entre $M$. canis et lui ; par conséquent, et jusqu'à preuve du contraire, il semble donc plus sage de la considérer comme une bonne espèce.

(21) Apportent peut-être une justification complémentaire à ce point de vue, un certain nombre d'observations qui montrent que la distinction entre $M$. audouini et $M$. canis n'est pas toujours aussi tranchée qu'on le présente à l'accoutumée : c'est ainsi que Catanei $(1939 c)$ a déterminé chez le cobaye une teigne expérimentale par inoculation d'une souche de $M$. audouini de type tardum de Sabouraud (1910) qu'il avait isolé d'une microsporie du Soudan Français ; que Kaplan et Georg (1957) ont publié récemment le cas d'un chien contaminé par deux enfants porteurs d'une teigne à $\boldsymbol{M}$. audouini, et que Vogei et Timpe (1957) ont vu le même parasite à l'origine d'une teigne spontanée d'un cobaye. D'autre part, Hare, en 1952, a isolé chez un enfant de Grande-Bretagne une souche de $M$. audouini qui produisait de nombreuses macroconidies. Avant lui, Scully et Kligman (1951) avaient cultivé, à partir cies lésions cutanées d'un singe, un dermatophyte qui ressemblait à $M$. audouini mais produisait de nombreuses macroconidies typiquement constituées.

(22) $M$. audouini n'a été isolé que de sujets de race blanche. 
(Sénégal, Angola, Afrique du Sud) (23) ; M. obesum (Dahomey, Togo, Cameroun). En apparence, la situation est relativement simple. On se trouve en présence d'une part de $M$. audouini et d'espèces satellites ( $M$. langeroni, $M$. rivalieri, $M$. catanei $(24)$, toutes très vraisemblablement anthropophiles), et, d'autre part, d'une unique espèce zoophile, $M$. canis, dont $M$. obesum ne constituerait qu'un synonyme. Il est cependant utile de préciser un certain nombre de faits :

$1^{\circ}$ Les deux souches de $M$. audouini isolées par Courmont en 1896 sur des enfants sénégalais différaient des souches classiques par la vivacité de leur culture. Nous-mêmes avions intitulé $M$. $a u$ douini cinq souches en provenance de Dakar et n'avons rectifié notre diagnostic (M. canis) qu'après examens microscopiques répétés et surtout après cultures sur riz. Les souches isolées à Lagos (Nigéria) par Clarke et Walker (1953) ne correspondaient pas aux souches classiques, mais étaient, suivant ces auteurs, très semblables à la souche de Hare (1952), à laquelle nous avons fait allusion plus haut (note 21, p. 720).

$2^{\circ}$ La mise en synonymie de $M$. obesum et $M$. canis par Conant (1945) est postérieure à la publication des travaux de Catanéi (1939). Or, nous ignorons si Catanéi aurait accepté d'identifier à M. canis les souches isolées par lui au Dahomey, au Togo et au Cameroun.

$3^{\circ}$ Catanéi (1939) a dénommé $M$. obesum les sept cultures de Microsporum provenant de prélèvements de tondantes microsporiques récoltées à Yaoundé. Les 31 souches de Microsporum étudiées par nous provenaient de cette ville et de ses environs ; elles étaient toutes très semblables, et nous les avons considérées comme une variété locale de $M$. audouini. En tout cas, elles étaient nettement plus proches de $M$. audouini que de $M$. canis. Des travaux en cours (en collaboration avec G. Duc) nous permettent d'ailleurs de confir-

(23) $M$. canis a été isolé chez des sujets de race blanche et de race noire.

(24) M. rivalieri a été décrit par Vanbreuseghem en 1951 à partir d'une souche unique. Cette souche atypique ressemblait macroscopiquement à $T$. tonsurans et avait la morphologie microscopique de $M$. audouini. $M$. catanei a été nommé par le même auteur (avec Gatti en 1955), mais n'a pas encore été décrit. Vanbreuseghem a eu l'amabilité de préciser, dans une lettre à l'un de nous, que ce champignon est très voisin de $M$. langeroni. Quant à cette dernière espèce, son intérêt est beaucoup plus considérable, car Vanbreuseghem l'a isolée de multiples fois au Congo Belge. Il diffère de. M. audouini principalement par la production d'un pigment rose très vif, parfois rouge groseille, qui diffuse dans la gélose. Ceci ne nous paraît toutefois pas justifier une nouvelle distinction spécifique, d'autant que certaines souches que nous avons isolées au Cameroun, par exemple, ressemblent fort à $M$. Iangeroni, mais à ce pigment près. Il est raisonnable de penser que tous ces champignons n'expriment que des variétés géographiques ou des formes culturales de $\boldsymbol{M}$. audouini. 
mer (cultures entreprises sur riz et inoculation aux cobayes) leur affinité à $M$. audouini. Faut-il admettre que nous ayons eu en mains des souches différentes de celles de Catanéi ou s'agit-il d'une différence d'interprétation à propos d'un même matériel ?

$4^{\circ}$ Cependant, au Togo, Catanéi (1939), sur 20 prélèvements de teignes microsporiques, porte 14 fois la diagnose de $M$. obesum et 6 fois celle de $M$. audouini. Cet auteur a donc été pour ce territoire en présence de deux types de colonies suffisamment distincts pour qu'il n'ait pas été tenté de les confondre, ce qui prend un intérêt tout particulier en raison de la très grande compétence de Catanéi. Rappelons cependant qu'en Haute-Volta, nous avons isolé quelques souches de culture difficile et de développement relativement pauvre qui, macroscopiquement, différaient nettement des autres, mais non microscopiquement, et que Catanéi $(1939 c)$ lui-même a observé des différences notables dans l'aspect des colonies de $M$. obesum, dans le nombre des fuseaux et dans le moment de leur apparition.

Il s'agit donc en définitive de savoir s'il existe effectivement en Afrique Noire, outre $M$. audouini et ses variantes locales, une ou des espèces différentes identiques ou affines à $M$. canis. La présence de cette dernière espèce paraît indiscutable en Afrique du Sud; Lurie a eu l'obligeance de nous préciser (lettre du 21 avril 1958) que les souches qu'il a isolées étaient typiques et que, dans de nombreux cas, on relevait la présence d'un chien ou d'un chat "galeux » dans la maison des enfants parasités. Mais la population de l'Afrique du Sud est très particulière, car l'apport européen y a été considérable. En Angola, Salazar Leite a posé cette diagnose trois fois ; malheureusement, elle n'est accompagnée d'aucun commentaire. Il est done bien difficile dans l'état actuel de nos connaissances d'arriver à une conclusion valable. Seule, une étude approfondie et systématique des souches isolées en Afrique Noire, ainsi qu'une collaboration étroite avec quelques confrères médecins-vétérinaires africains, effectuant de façon parallèle une enquête concernant les teignes des animaux domestiques dans les territoires considérés, permettraient peut-être d'apporter une solution définitive à un problème de taxonomie dont l'intérêt théorique est accru par son retentissement épidémiologique.

\section{3) Remarques sur les associations mycologigues}

Nous y avons déjà fait allusion plus haut à propos des teignes mixtes, puisque celles-ci ne furent révélées que par la culture des prélèvements effectués. Mais, indépendamment des cas où sont 
associés sur une même tête un agent de Microsporie et un agent de Trichophytie, on isole parfois deux dermatophytes déterminant une même forme clinique de teigne. Les associations mycologiques sont encore mal connues et celles qui furent signalées en Afrique Noire sont dues à Catanéi (1939 a) pour la Guinée Française, à Vanbreuseghem (1950) pour le Congo Belge, à Clarke et Walker (1953) pour le Nigéria, à Cochet et coll. (1957) pour le Cameroun, à nous-mêmes pour la Haute-Volta.

Ont été cultivées jusqu'ici :

T. soudanense $+T$. gourvili.

$T$. soudanense $+T$. yaoundei.

$T$. soudanense $+T$. ferrugineum.

T. soudanense $+M$. audouini.

$T$. gourvili $+T$. ferrugineum.

$T$. yaoundei $+T$. ferrugineum.

T. yaoundei $+M$. audouini.

$T$. violaceum $+T$. ferrugineum.

T. violaceum + M. audouini.

Nous avons rassemblé dans le tableau $\mathrm{V}$ ces diverses associations en fonction des territoires où elles ont été observées. Il est très vraisemblable que l'on en découvrira d'autres au fur et à mesure que seront multipliés les ensemencements de matériel en provenance de l'Afrique Noire. Jusqu'ici, il ne semble pas qu'aient été découverts simultanément deux agents de Microspories ( $T$. ferrugineum et $M$. audouini, par exemple).

\section{RÉsumÉ}

Sur 171 prélèvements positifs de cheveux effectués chez des enfants de Haute-Volta, nous avons observé 148 Trichophyties $(86,54 \%), 21$ Microspories $(12,8 \%)$ et deux tondantes mixtes, mais aucun Favus. Nous avons utilisé 109 souches qui appartiennent aux espèces suivantes : Trichophyton gourvili (64), T. soudanense (23), T. rubrum (2) et T. tonsurans (1), agents des Trichophyties; T. ferrugineum (4) et Microsporum audouini (15), agents des Microspories.

Ce travail a été l'occasion d'une revue générale des publications concernant les dermatophyties et les dermatophytes en Afrique Noire. D'une façon générale, il a d'abord mis en évidence les lacunes considérables de notre documentation actuelle et les divergen- 


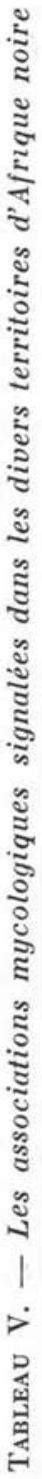

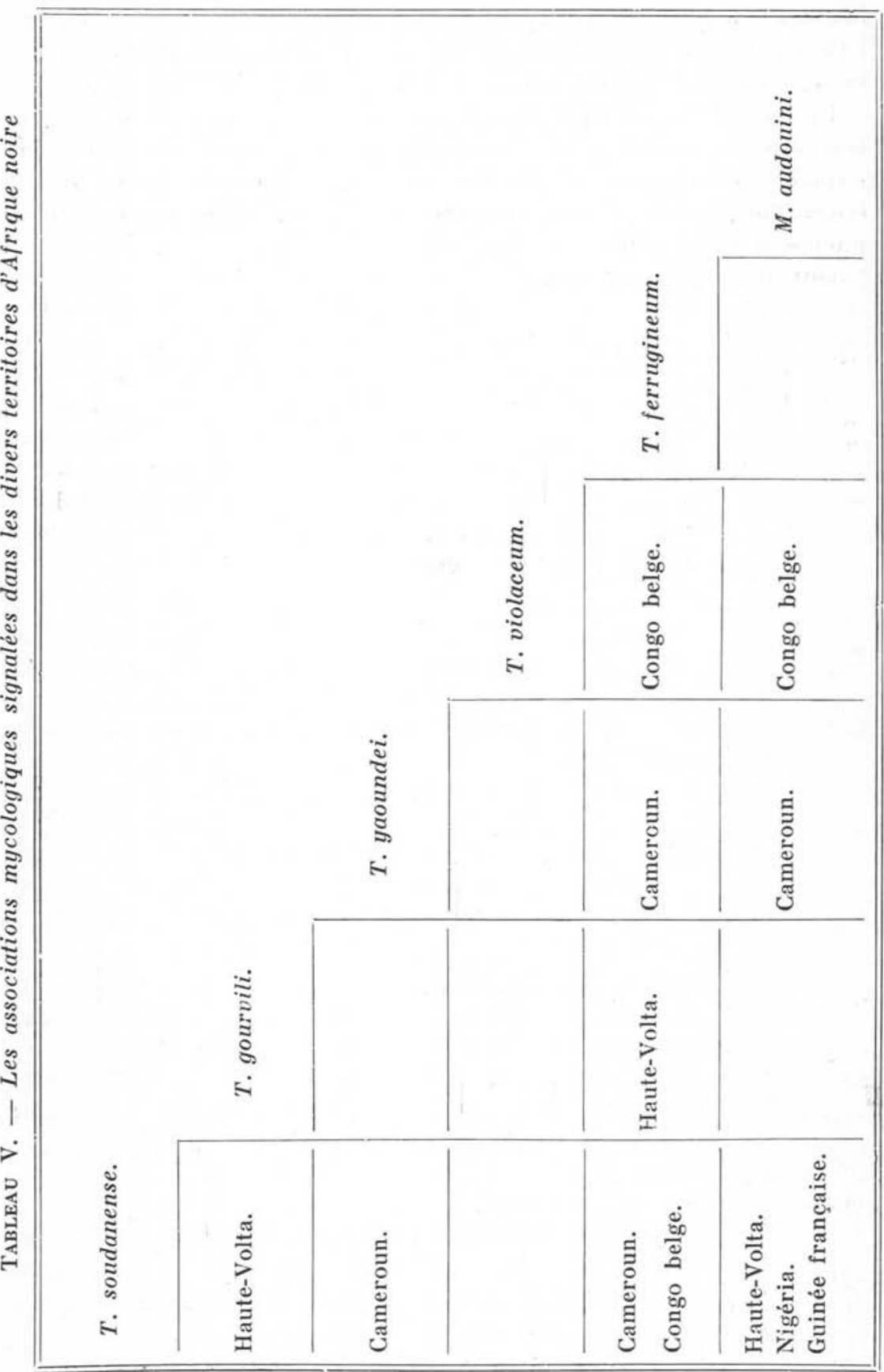


ces des conceptions taxonomiques des auteurs. Nous nous sommes efforcés d'unifier la nomenclature après avoir discuté les diagnoses lorsque celles-ci nous semblaient critiquables.

Le tour d'horizon que nous avons effectué confirme la rareté du Favus dans la plupart des territoires de population noire et met l'accent sur les erreurs possibles dans son diagnostic. Après avoir étudié la répartition des tondantes et les variations de leur fréquence relative suivant les lieux considérés, nous avons exposé la localisation géographique des principaux dermatophytes signalés jusqu'ici par les auteurs. Nous avons enfin pris conscience des difficultés de diagnose des Microsporum africains ; macroscopiquement et microscopiquement assez polymorphes, peu caractérisés dans la règle, leurs affinités pour $M$. audouini ou $M$. canis sont difficiles à préciser sans une étude préalable particulièrement approfondie; dans le cadre de ces travaux sur les Microsporum, nous suggérons que soit réalisée chaque fois que possible, parallèlement aux recherches intéressant l'homme, une enquête sur les teignes des animaux familiers, réservoirs éventuels de virus.

\section{BibLIOGRAPHIE}

Brumpt (E.), 1949. - Précis de Parasitologie, $6^{\circ}$ édition, Paris, Masson et $\mathrm{C}^{\prime e}$, édit.

CataneI (A.), 1933, - Etudes sur les teignes. Arch. Inst. Pasteur d'Algérie, XI, p. 267.

Catanei (A.), 1939 a. - Premiers résultats d'une étude des teignes dans les colonies françaises. Bull. Soc. Path, Exot, XXXII, p. 247.

CataneI (A.), $1939 \mathrm{~b}$. - Sur la répartition des différentes espèces de champignons des teignes de l'homme en Afrique. Arch. Inst. Past. d'Algérie, XVII, p. 613.

Catanei (A.), 1939 c. - Etude des teignes du cuir chevelu dans les colonies françaises. Arch. Inst. Pasteur d'Algérie, XVII, p. 47.

Chalmers (A. J.) et Marshals. (A.), 1914. - Tinea capitis tropicalis in the Anglo-Egyptian Sudan. Journ. Trop. Med. and Hyg., XVII, p. 257.

Cl.arke (G. H. V.) et Walker (J.), 1953. - Superficial fungus infections in Nigeria. Journ. Trop. Med, and Hyg., LVI, p. 117.

Cochet (G.) et Dony-Dubors (M.), 1957. - Contribution à la connaissance des teignes infantiles du Cameroun (Note préliminaire). Semaine des Hopitaux, XXXIII, p. 2980.

Cochet (G.), Doby-Dubots (M.), Deblock (S.), Doby (J. M.) et Varva (C.), 1957. Contribution à la connaissance des teignes infantiles du Cameroun. Annales de Parasit. hum. et comp., XXXII, p. 580.

Conant (N. F.), Smith (D. T.), Baker (R. D.), Callaway (J. L.) et Martin (D. D ), 1945 (1 $^{\text {re édit.) }}$ et 1954 (2 édit.). - Manual of Clinical Mycology, Philadelphie, W. B. Saunders edit.

Coudert (J.), 1955, - Mycologie médicale, Paris, Masson édit. 
Courmont (P.), 1896. - Etude clinique et expérimentale sur quelques types nouveaux de teignes exotiques. Arch. de Méd. Trop., VIII, p. 700.

Coutelen (F.), Cochet (G.), Biguet (J.), Mullet (S.), Doby-Dubois (M.) et Deblock (S.), 1956. - Contribution à la connaissance épidémiologique et mycologique des teignes infantiles de Tunisie. Ann. de Parasit. hum. et comp., XXXI, p. 449.

DE Mello (F.), 1917-1918. - Trichophyton viannei n. sp. The infecting agent in a case of dermatomycosis. The Indian Journ. of Med. Research, V, p. 222 .

De Melio (F.) et Paes (S.), 1923. - Sur un Trichophyton à culture faviforme, agent d'une teigne du cuir chevelu. Rev. Méd. de Angola, IV, p. 505.

Dodge (C. W.), 1936. - Medical Mycology, London, H. Kimpton edit.

Doupagne (P.), 1957. - Athlete's foot au Congo Belge chez les militaires autochtones. Ann. Soc. Belge de Méd. Trop., XXXVII, p.. 245.

Eмmoss (C. W.), 1934. - Dermatophytes. Natural grouping based on the form of the spores and accessory organs. Archiv. Derm. Syphil., XXX, p. 337.

Georg (L. K.), 1956. - Studies on Trichophyton tonsurans. I. The taxonomy of T. tonsurans. Mycologia, XLVIII, p. 65.

HARE (P. J.), 1952. - A strain of Microsporum audouini producing numerous macroconidia on culture. The British Journ. of Dermatology, LXIV, p. 236.

Hazes (H.), 1914. - Personal observations upon skin diseases in the american negro. Journ. Cutan. Dis., XXXII, p. 705.

Hazes (H.), 1935. - Syphilis and skin diseases in the american negro : personal observations. Archiv. Derm. and Syph., XXXI, p. 316.

Huriez (C.), Biguet (J.), Cochet (G.), Mullet (S.) et Doby-Dubois (M.), 1957. Epidémiologie des teignes infantiles dans le Nord de la France depuis 1946. Semaine des Hopitaux de Paris, XXXIII, p. 2046.

Jeanselme (E.), 1904. - Cours de Dermatologie Exotique, Paris, Masson et $\mathrm{C}^{\text {io }}$ éd.

Joyeux (C.), 1912. - Sur le Trichophyton soudanense n. sp. C.R. Soc. Biol., LXXIII, p. 15.

Kaplan (W.) et Geong (L. K.), 1957. - Isolation of Microsporum audouini from a dog. The Journ. of Investigative Dermatology, XXVIII, p. 313.

Langeron (M.) et Vanbreuseghem (R.), 1952. - Précis de Mycologie, Paris, Masson et $\mathrm{C}^{\text {je }}$ édit.

Lapidus (R.), 1950. - Les teignes en Tripolitaine, thèse Doct. Méd. Paris.

Loewenthal (L. S. A.), 1939. - Diseases of the skin in the negroes. Journ, of Trop. Med, and Hyg., XLI, p. 189, et XLII, p. 20.

LUrie (H. 1.), 1955. - Fungal diseases in South-Africa. South African Med. Journ., XXIX, p. 186.

Mitchell (J. A.) et Robentson (G. W.), 1924. - « Witkop 》 or « Dikwakwadi 》 in Bechuanaland natives. Report of investigation. South African Med. Journ., XX, p. 122.

PaEs (S.), 1923. - Nouvelle contribution à l'étude des dermatomycoses d'Angola. $I^{e r}$ Congrès de Méd. Trop. de l'Afrique Occidentale, Loanda.

Petges (G.) et Lecoulant (P.), 1937. - Teigne d'origine africaine (région du Tehad) chez un enfant de 22 mois, à parasite rappelant le Microsporum ferrugineum Ota, 1921. Essai d'identification. Ann. Dermat. et Syph., VIII, p. 447.

FIJPER (A.), 1918. - Tinea imbricata in South Africa. Journ. of Trop. Med. and Hyg., XXI, p. 45.

Pondexter (H. A.), 1949. - A laboratory epidemiological study of certain infection diseases in Liberia. Amer. Journ. of Trop. Med., XXIX, p. 435. 
Ranque (J.) et Temise (P.), 1957. - Etat actuel des dermatomycoses dans la région marseiliaise. Semaine des Hopitaux de Paris, XXXIII, p. 2290.

Rodhain (J.), 1943. - Quelques données au sujet des teignes de Mayumbe. Ann. Soc. Belge de Méd. Trop., XXIII, p. 63.

Rophais (J.), 1943. - Documents complémentaires au sujet des teignes au Mayumbe. Ann. Soc. Belge de Méd. Trop., XXIII, p. 257.

Sabouraud (R.), 1894. - Les Trichophyties humaines, Paris, Rueff et $\mathrm{C}^{\text {le }}$ édit.

Sabovraud (R.), 1910. - Maladie du cuir chevelu. III. Les maladies cryptogamiques. Les Teignes. Paris, Masson et $\mathrm{C}^{10}$ édit.

Salanoue-Ipin (H.), 1919. - In Loewenthal, 1939.

Salazar Leite (A.), 1949. - Aspecto da Micologia medica em Angola. Journal de Medico, XIV, p. 199.

Salazar Letre (A.) et Bastos da Luz (I. V.), 1950. - Estudos sobre os agentes causais de tinhas em nativos africanos de raça negra. Instit. de Med. Trop. Dermatologia e Mycologia Tropicais. II. Congresso Luso-Espanhol de Dermatologia, Lisboa.

Salazar Leite (A.), Bastos da Luz (I. V.) et De Meira (M. T. V.), 1947. - Relatorio de Missão Medica do instituto de Medicina Tropical a Angola em 1945. Anais de Inst. Med. Trop., IV, p. 465.

Salazar Leite (A.) et Re (L.), 1955. - Contribution à l'étude ethnologique des populations africaines. Arch. Inst. Pasteur d'Algérie, XXXIII, p. 344.

Scully (J. P.) et Kligman (A. M.), 1951. - Coincident infection of a humain and an anthropoid with Microsporum audouini. Arch. Dermat. and Syph., LXIV, p. 495.

Segretain (G.), 1947. - Sur un Trichophyton rubrum d'origine africaine. Ann. de l'Inst. Pasteur, LXXIII, p. 395.

Sмrth (E. C.), 1932. - An Atlas of skin Diseases in the Tropies, London.

VANBreuseghem (R.), 1950. - Diagnose et systématique des Dermatophytes. Contribution à la connaissance des teignes au Congo Belge. Ann. Soc. Beige de Méd. Trop., XXX, p. 865.

Vanbreuseghem (R.), 1951. - Sur un nouveau dermatophyte isolé au Congo Belge, Sabouraudites rivalieri n. sp. Arch. belges de Derm. et Syph., VII, p. 109.

Vanbreuseghem (R.), 1955. - Le Congo Belge et la mycologie médicale. Mémoires Acad. Roy. Sc. Col., I, p. 1 (*).

VANBREUSEGHEM (R.), 1957. - Tinea capitis and african histoplasmosis in the belgian Congo. Trans. of the New York Acad. of Sciences, série II, XIX, p. 622 .

Vanbreuseghem (R.) et Gatti (F.), 1955. - Enquète sur l'incidence des teignes du cuir chevelu chez les indigènes du Congo Belge. Ann. Soc. Belge de Méd. Trop., XXXv, p. 805.

Vogel (R. A.) et TJMPe (A. M.), 1952. - Spontaneous Microsporum audouini infection in a guinea pig. The Journ. of Investigat. Dermat., XXVIII, p. 311 .

Ziemann (H.) et SKLAReck (B.), 1932. - Jadassohn's Handbuch der Haut und Geschlechts-Krankheiten, XII, p. 499, in LoEwenthaL, 1939.

(*) On trouvera dans cette publication toutes les références des Travaux de Vanbreuseghem antérieurs à 1955 et ayant trait à la pathologie mycologique du Congo Belge.

(Travail de la Chaire de Zoologie et Parasitologie de la Faculté de Médecine de Lille. Professeur : J. Biguet) 